\title{
Gut microbiota-stimulated cathepsin K secretion mediates TLR4-dependent M2 macrophage polarization and promotes tumor metastasis in colorectal cancer
}

\author{
Rui $\mathrm{Li}^{1,2} \cdot$ Rui Zhou ${ }^{1,2} \cdot$ Hui Wang ${ }^{3} \cdot$ Weidong $\mathrm{Li}^{3} \cdot$ Mingxin $\mathrm{Pan}^{4} \cdot$ Xueqing Yao $^{5} \cdot$ Wanqi Zhan $^{2} \cdot$ Shibin Yang $^{6} \cdot$ \\ Lijun $\mathrm{Xu}^{2} \cdot$ Yanqing Ding ${ }^{1,2} \cdot$ Liang Zhao $\mathbb{1}^{1,2}$
}

Received: 29 October 2018 / Revised: 3 February 2019 / Accepted: 19 February 2019 / Published online: 8 March 2019

(c) ADMC Associazione Differenziamento e Morte Cellulare 2019

\begin{abstract}
Metastasis is a complex process that requires the interaction between tumor cells and their microenvironment. As an important regulator of intestinal microenvironment, gut microbiota plays a significant role in the initiation and progression of colorectal cancer (CRC), but the underlying mechanisms remain elusive. In this study, a metastasis-related secretory protein cathepsin K (CTSK) was identified as a vital mediator between the imbalance of intestinal microbiota and CRC metastasis. We implanted MC38 cells into the cecal mesentry of antibiotic-treated mice with intragastrically administration of $E$. coli to mimic gut microbiota imbalance. The bigger primary tumors and more liver metastatic foci were detected in the E. coli group accompanied with high LPS secretion and CTSK overexpression compared with that in the control group. CTSK contributes to the aggressive phenotype of CRC cells both in vitro and in vivo. Silencing CTSK or administration of Odanacatib, a CTSK-specific inhibitor, totally abolished the CTSK-enhanced migration and motility of CRC cells. Interestingly, the tumorsecreted CTSK could bind to toll-like receptor 4 (TLR4) to stimulate the M2 polarization of tumor-associated macrophages (TAMs) via an mTOR-dependent pathway. Recombinant CTSK could neither stimulate CRC growth and metastasis, nor induce M2 macrophage polarization in TRL4 ${ }^{-1-}$ mice. Meanwhile, CTSK could stimulate the secretion of cytokines by M2 TAMs including IL10 and IL17, which, in turn, promote the invasion and metastasis of CRC cells through NFkB pathway. Clinically, overexpression of CTSK in human CRC tissues is always accompanied with high M2 TAMs in the stroma, and correlated with CRC metastasis and poor prognosis. Our current research identified CTSK as a mediator between the imbalance of gut microbiota and CRC metastasis. More importantly, we illustrated a CTSK-mediated-positive feedback loop between CRC cells and TAMs during metastasis, prompting CTSK as a novel predictive biomarker and feasible therapeutic target for CRC.
\end{abstract}

These authors contributed equally: Rui Li, Rui Zhou, Hui Wang

Edited by R. Johnstone

Supplementary information The online version of this article (https:// doi.org/10.1038/s41418-019-0312-y) contains supplementary material, which is available to authorized users.

Yanqing Ding

dyqgz@126.com

$\triangle$ Liang Zhao

liangsmu@foxmail.com

1 Department of Pathology, Nanfang Hospital, Southern Medical University, Guangzhou, Guangdong, China

2 Department of Pathology, School of Basic Medical Sciences, Southern Medical University, Guangzhou, Guangdong, China

\section{Introduction}

Colorectal cancer is the third most common malignancy and the second leading cause of cancer death worldwide [1]. Despite advances in the diagnosis and treatment of $\mathrm{CRC}$, metastasis remains the major cause of the

3 Department of Medical Oncology, Affiliated Tumor Hospital of Guangzhou Medical University, Guangzhou, China

4 Second Department of Hepatobiliary Surgery, Zhujiang Hospital, Southern Medical University, Guangzhou, China

5 Department of General Surgery, Guangdong General Hospital, Guangdong Academy of Medical Science, Guangzhou, China

6 Gastrointestinal Surgical Center, First Affiliated Hospital of Sun Yat-sen University, Guangzhou, China 
high mortality and poor prognosis. Metastasis is a multistage process modulated by non-malignant cells from the tumor microenvironment that continuously promotes the growth, migration, and invasion of host tumor cells [2]. Intestine obtains the largest microbial colonization in human body, which has been demonstrated contributing to the occurrence [3], development [4], and immunotherapy [5] of colorectal cancer. Imbalance of intestinal microbiota, termed as intestinal dysbiosis, triggers the secretion of cytokines by immune cells like lymphocytes and macrophages to affect the self-repair of colonic epithelial cells [6]. Moreover, intestinal dysbiosis also increases the release of endotoxins, which could bind to pattern recognition receptors, especially Toll-like receptors and Nod-like receptors, to promote tumor cell proliferation, invasion, and metastasis [7]. Although large amounts of studies focus on the interaction between microbiota and inflammatory cells in CRC development and progression, the underlying mechanisms are still controversial.

Cathepsin K (CTSK), a lysosomal cysteine protease belongs to the peptidase protein $\mathrm{C} 1$ family, is predominantly expressed in osteoclasts involving in bone remodeling and resorption [8]. Human CTSK participates in a considerable number of physiological processes, including MHC-II-mediated antigen presentation, bone remodeling, keratinocyte differentiation, and prohormone activation [9]. CTSK could also be detected in several cancer types including oral squamous cell carcinoma [10], breast cancer [11], ovarian cancer [12], and glioblastoma [13]. Despite its well-known function in cleaving and activating MM9 to degrade and remodel the tumor extracellular matrix [14], tumor-secreted CTSK might also interact with stromal cells in a paracrine manner. Overexpression of CTSK was detected in breast cancer tissues, but only the high stromal CTSK expression was correlated with the low ER and PR expression and the poor clinical prognosis [15]. Moreover, the concentration of serum CTSK was significantly increased in ovarian cancer patients, prompting the possibility of CTSK as a novel clinical diagnostic biomarker for ovarian cancer [12]. However, the expression of CTSK in colorectal cancer and its role in CRC growth and progression is totally unknown.

In this study, we identified CTSK as a metastasis-related protein upregulated by imbalance of microbiota. The influences of CTSK on the aggressive phenotypes of CRC were further confirmed. Moreover, the effects of tumorsecreted CTSK on M2 polarization of TAMs were also determined, as well as the corresponding cytokines they released. The current study will uncover the linkage between intestinal microbiota and CRC metastasis, and provide a novel predictive biomarker and feasible therapeutic target for CRC.

\section{Materials and methods}

\section{Cell culture}

The normal human colon epithelial cell line FHC, NCM460, and colorectal cancer cell lines including SW480, SW620, HCT116, LS174T, HT29, and LOVO were obtained from the Cell Bank of the Chinese Academy of Sciences (Shanghai, China) and maintained as previously described [16]. All the cells were cultured in RPMI-1640 (Hyclone, Logan, UT, USA) supplemented with $10 \%$ fetal bovine serum (Gibco-BRL, Invitrogen, Paisley, UK) at $37^{\circ}$ $\mathrm{C}$ with a humidity of $5 \% \mathrm{CO}_{2}$.

\section{Co-incubation of Escherichia coli (E. coli) and CRC cells}

Escherichia coli (E. coli) were grown in Luria-Bertani (LB) broth at $37^{\circ} \mathrm{C}$ overnight to mid-log phase. Concentration of bacteria was adjusted based on the optical density reading at $600 \mathrm{~nm}$ analyzed by NanoDrop ND-2000. Bacteria were diluted to 1:10 with appropriate medium prior to co-culture with CRC cells [17]. Briefly, SW480 cells w/o treatment of PMA-primed macrophage-like U937 cells were seeded into 12-well lumox plates (Vivascience, Gloucestershire, UK) and grown to $70 \%$ confluence before co-cultured with $E$. coli at $10^{6} \mathrm{CFU} / \mathrm{ml}$ in RPMI-1640 supplemented with $10 \%$ fetal bovine serum for $6 \mathrm{~h}$. E. coli were allowed to infect the monolayers of CRC cells. All extracellular bacteria were killed by gentamicin $(500 \mu \mathrm{g} / \mathrm{ml}$ for $20 \mathrm{~min})$ and the dead bacteria were removed by extensive washing with phosphate buffer saline (PBS) [17].

\section{Orthotopic implantation of colorectal cancer cells in antibiotic-treated mice}

Approximately, $1 \times 10^{6} \mathrm{MC} 38$ cells were injected into the cecal mesentry of female C57 mice at 6 weeks of age. After 10 days, gut microbiota of the tumor-bearing mice were removed by intragastrically antibiotics treatment (A mixture of neomycin, penicillin metronidazole, and vancomycin) for 5 days. A $100 \mu \mathrm{l} E$. coli at a concentration of $1 \times 10^{8} \mathrm{CFU} / \mathrm{ml}$ in PBS were administrated to mice once daily for 3 days by a gavage needle 2 days after antibiotics treatment. 16s RNA sequencing of mice feces was applied to confirm the successfully established of the animal model. The mice were sacrificed and tumors were harvested 5 days after $E$. coli inoculation, which makes the total experimental period of 24 days [18].

\section{Tumor tissue samples}

Fresh primary CRC specimens with paired noncancerous colorectal tissues were obtained from the Tumor Tissue 
Bank of Nanfang Hospital. In each case, a diagnosis of primary CRC had been made, and the patient had undergone elective surgery for CRC in Nanfang Hospital between 2015 and 2018. The Ethics Committee of Southern Medical University approved the study and all aspects of the study comply with the Declaration of Helsinki.

\section{Animals}

All animal experiments were approved by the Institutional Animal Care and Use Committee of Southern Medical University (Guangzhou, China). Female BALB/c nude mice (3-5 weeks, 14-16 g) obtained from Nanfang Medical University Experimental Animal Center were housed in SPF animal room and had free access to sterilized food and water. Mice were divided into five groups: (1) control group injected with shNC-SW480 cells; (2) knock down group injected with shCTSK-SW480 cells; (3) control group injected with LV-ctrl-RKO cells; (4) overexpression group injected with LV-CTSK-RKO cells; and (5) medication group injected with LV-CTSK-RKO cells were orally administered pharmacologic grade Odanacatib (MK-0822; $3.606 \mathrm{mg} \mathrm{kg}^{-1}$ per week, dissolved in dimethyl sulfoxide (DMSO)) from 1 week before the injection to the end of sample harvest. The Odanacatib dose was chosen according to the treatment of humans and converted to an equivalent dose for mice. The control mice were orally administered DMSO.

For the construction of colon cancer xenograft model, $5 \times 10^{6}$ cells were injected into the mammary fat pad of each nude mouse. After 4 weeks, mice were sacrificed by cervical dislocation and the xenograft tumors were quickly harvested for histological study. The tumor volume was calculated according to the formula: Volume $\left(\mathrm{mm}^{3}\right)=$ width $^{2}\left(\mathrm{~mm}^{2}\right) \times$ length $(\mathrm{mm}) / 2$. The total 75 mice were used in three independent experiments.

For the construction of metastasis model, $2 \times 10^{6}$ cells $(100 \mu \mathrm{l})$ were injected into the left or right tail vein of female BALB/c nude mice. After 6 weeks, the nude mice were dissected; lung and liver tissues were collected (seven mice per group).

C57BL/10ScNJNju $\left(\mathrm{TLR}^{-1-}{ }^{-}\right.$) mice $(\mathrm{ScNJ})$ were purchased from Nanjing Biomedical Research Institute. Blood was extracted from mice tail top and reverse transcription polymerase chain reaction (RT-PCR) experiments were performed to confirm the knockout of TLR4. MC38 cells $\left(1 \times 10^{6}\right)$ were injected into the cecal mesentry of WT and ScNJ mice at 6 weeks of age. Five days after the orthotopic implantation of MC38 cells, recombinant human CTSK protein (rCTSK, Bio Vision, 1026-10; $1 \mathrm{\mu g} \mathrm{g}^{-1}$ body weight) or saline were injected into the peritoneal cavity of mice once a week, respectively. One week later, mice were injected every 3 days with rCTSK or saline, which makes the total experimental period of 21 days [19].

\section{U937 monocyte transwell assay}

PMA-primed macrophage-like U937 cells were used for transwell assays. U937 cells obtained from ATCC were cultured in the suggested medium for $24 \mathrm{~h}$ before primed with $10 \mathrm{nM}$ phorbol 12-myristate 13-acetate (PMA, Sigma) for $48 \mathrm{~h}$. Transwell assays assessing cell migration or invasion were performed on 24-well plate with inserts (BD Biosciences) according to the manufacturer's instruction. Primed U937 cells were seeded in the bottom chamber at a concentration of $5 \times 10^{5}$ cells $/ \mathrm{ml}$ and the $\mathrm{CRC}$ cells in the upper chamber were allowed to migrate or invade for $12-24 \mathrm{~h}$ before fixation for crystal purple staining.

Recombinant CTSK protein was added into the culture medium of monocyte-derived macrophages and harvested after $24 \mathrm{~h}$. Conditional medium (CM) used for western blot analysis or the cell migration transwell assay was obtained from culturing CRC cells in RPMI/ 1640 media with $0.1 \%$ fetal bovine serum for $24 \mathrm{~h}$ and concentrated via ultrafiltration (Millipore, $15 \mathrm{ml}$ 10KD).

\section{Isolation and treatment of mouse peritoneal macrophages}

Peritoneal macrophages were isolated from healthy female C57BL/6 mice (6-8-weeks-old) as previously described [20]. Briefly, $1 \mathrm{ml} 4 \%$ starch beef extract broth was injected to mice intraperitoneally 3 days prior to the harvest of peritoneal microphages. Mice were sacrificed and macrophages in the peritoneal cavity were lavaged using $5 \mathrm{ml} 1640$ medium. Peritoneal fluid was aspirated from peritoneum and centrifuged $(1000 \mathrm{rpm}$, Eppendorf 5810R) at $4{ }^{\circ} \mathrm{C}$ for $10 \mathrm{~min}$. Supernatant was discarded and the pellet was resuspended and washed with cold PBS for three times. To characterize the purity of isolated macrophages, cells were examined by flow cytometry $8 \mathrm{~h}$ after isolation.

\section{Western blot analysis}

The immunoblot analysis of cell lysates $(20-60 \mathrm{mg}$ ) in RIPA buffer was carried out for assessing protein expression in the presence of rabbit antibodies to CTSK (Abcam, 19027, 1:500), Phospho-Akt (CST, 4060, 1:1000), Phospho-mTOR (CST, 5536, 1:500), Phospho4E-BP1 (CST, 9456, 1:500), Phospho-NF-кB p65 (CST, 3033, 1:1000), Phospho-IKK $\alpha / \beta$ (CST, 2697, 1:1000), 

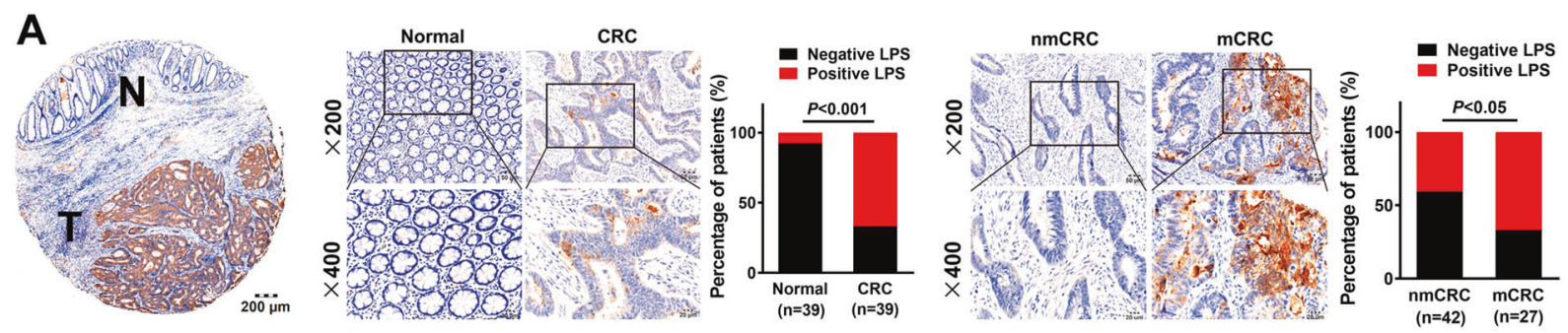

B
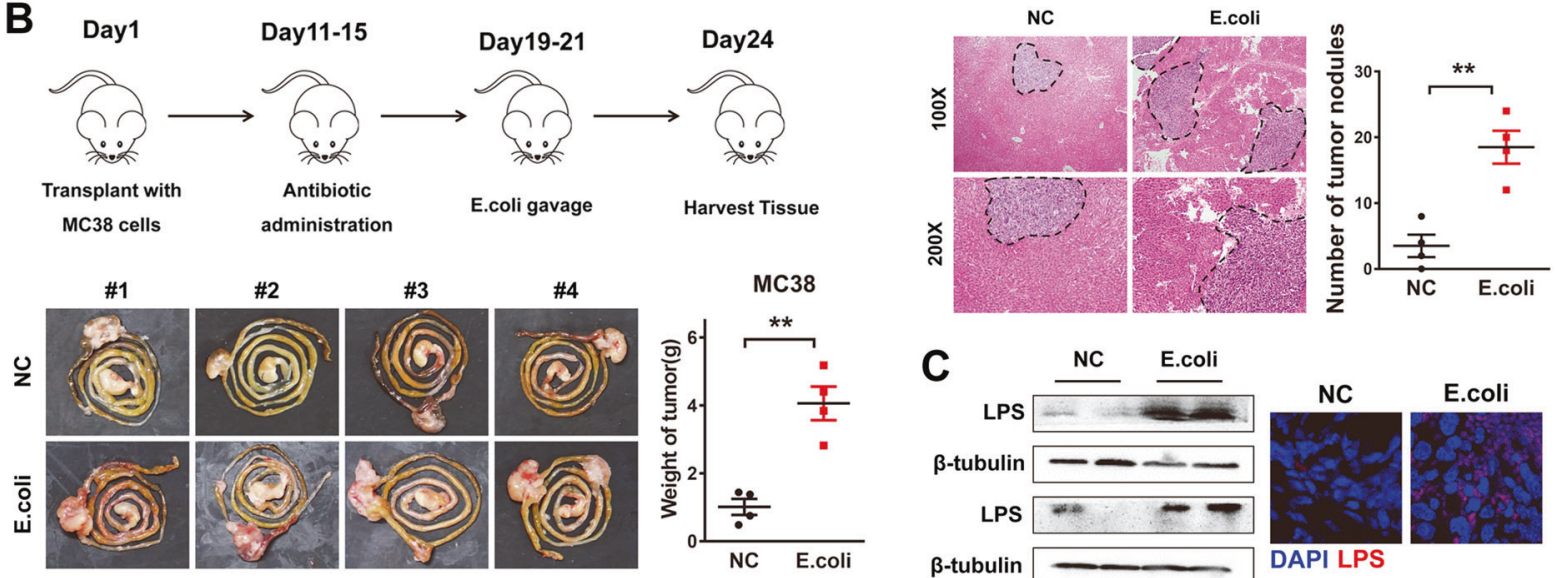

D

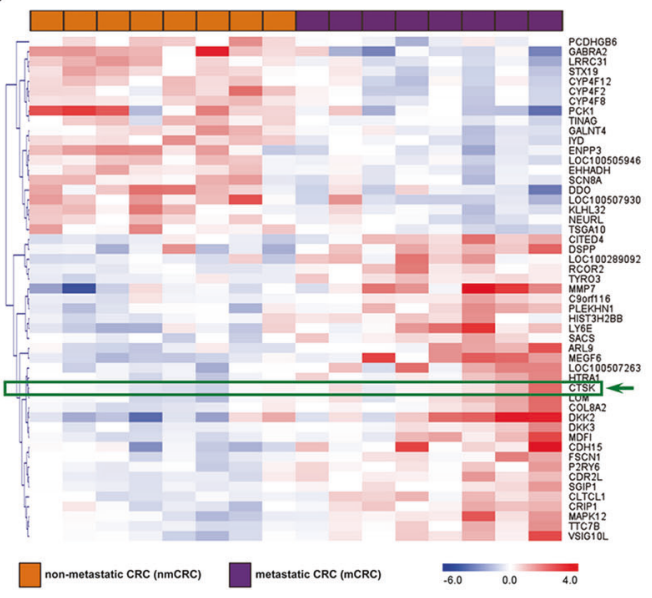

E
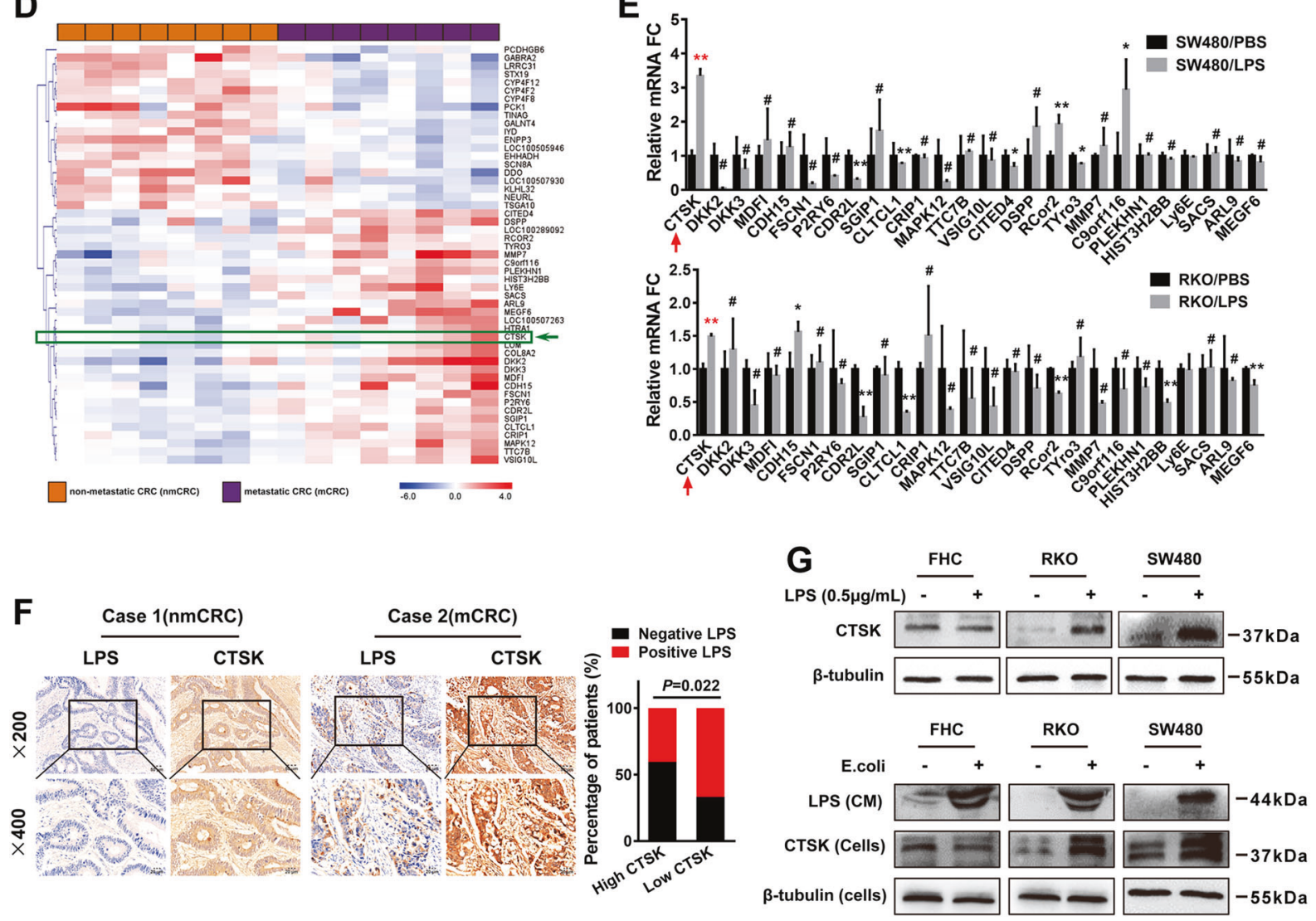

Phospho-IкB $\alpha$ (CST, 2895, 1:1000); mouse antibodies to CD206 (Santa Cruz, 34577, 1:300), CD68 (ZA, 0060, 1:200), TLR4 (Santa Cruz, 293072, 1:500), MyD88
(Santa Cruz, 74532, 1:500), $\beta$-tubulin (Abcam, 56676, 1:1000), IкB $\alpha$ (CST, 4814, 1:1000), and IKK- $\alpha$ (CST, 11930, 1:1000). 
Fig. 1 Imbalance of intestinal microbiota is associated with high CTSK expression and advanced progression of colorectal cancer. a Left panel: Immunohistochemistry (IHC) analysis of lipopolysaccharides (LPS) in colorectal cancer (CRC) tissues and adjacent nontumor tissues. Right panel: IHC analysis of LPS in CRC tissues w/o metastasis. b Upper left panel: Sketch map shows the orthotopic implantation of MC38 cells in antibiotic-treated mice w/o intragastrical administration of E. coli. Upper right panel: HE staining shows the hepatic metastasis foci in antibiotic-treated mice w/o intragastrical administration of $E$. coli. The scatter diagram on the right represents the number of tumor nodules. Lower panel: The primary tumor developed by implantation of MC38 cells in antibiotic-treated mice w/ o intragastrical administration of $E$. coli. c LPS expression in the primary tumors developed by implantation of MC38 cells in antibiotictreated mice w/o intragastrical administration of E. coli. d Heat map depicting the differentially expressed genes in eight pairs of human non-metastatic and metastatic CRC tissues. Red and blue indicate high and low expression of genes. e Real-time PCR analysis shows the expression of differentially expressed genes in response to LPS treatment in SW480 and RKO cell lines. f Representative images of IHC staining analyses of CTSK and LPS in non-metastatic and metastatic CRC tissues. $g$ Upper panel: Western blot analysis of CTSK in FHC, SW480 and RKO cell lines in response to LPS treatment. Lower panel: Western blot analysis of LPS releasing and CTSK in FHC, SW480, and RKO cells pretreated with E. coli

\section{Immunofluorescence}

Immunofluorescence staining was performed according to the standard protocol described previously [21]. Polyclonal rabbit primary antibody to CTSK (Abcam, 19027, 1:500), mouse antibodies to CD206 (Santa Cruz, 34577, 1:300), CD68 (ZA, 0060, 1:200), TLR4 (Santa Cruz, 293072, 1:500), HRP-conjugated secondary antibody and DAB staining kit (CWBIO, Beijing, China) were used in the experiment. Recombinant human IL4 and IL13 (PeproTech, $20 \mathrm{ng} / \mathrm{ml}$ ) were applied $24 \mathrm{~h}$ before cells were harvested.

\section{Immunohistochemistry (IHC)}

IHC was performed to investigate the expression of proteins in human colorectal cancer tissues. The sections were incubated overnight using primary antibodies against CTSK (Abcam, 19027, 1:500) and CD163 (Santa Cruz, 20066, 1:200) at $4{ }^{\circ} \mathrm{C}$. Mayer's hematoxylin was used as the purpose of nuclear counter staining. In this study, these slides were reviewed by two or three blind-folded pathologists. The IHC staining was scored semi quantitatively as 0 (no staining), 1 (weak staining, light yellow), 2 (moderate staining, yellowish brown), and 3 (strong staining, brown) based on the intensity of staining. The IHC score of 2 and 3 was considered as high expression (or overexpression), whereas $<2$ was regarded as low expression. The discrepancies $(<5 \%)$ were resolved by simultaneous reevaluation. The Pearson $\chi^{2}$ test was used to determine the significance of correlation.

\section{Statistical analysis}

Data were analyzed using SPSS statistic software version 19.0 (SPSS; Chicago, USA). The Student $t$-test and the oneway ANOVA test were carried out for RT-PCR. KaplanMeier plots were used to estimate the prognostic relevance of CTSK in univariate analysis. Multivariate analysis was performed by Cox proportional hazards test. Statistical significance was established at $P<0.05$.

\section{Results}

\section{Imbalance of intestinal microbiota is associated with high CTSK expression and advanced progression of colorectal cancer}

To identify the correlation between intestinal microbiota imbalance and CRC progression, we detected the releasing of lipopolysaccharide (LPS), the most common endotoxin brought by intestinal dysbiosis, in human CRC tissues. Positive LPS was detected in 7.7\% (3/39) of normal colorectal samples and a much higher rate was observed in $66.7 \%$ (27/39) of CRC samples (Fig. 1a, left panel, $P<$ 0.001 ). Moreover, positive LPS was detected in $40.5 \%$ (17/ 42) of non-metastatic CRC (nmCRC) tissues and a much higher rate was observed in $66.7 \%(18 / 27)$ of metastatic CRC (mCRC) tissues (Fig. 1a, right panel, $P<0.001$ ). To further confirm this relationship, we orthotopically implanted MC38 cells to antibiotic-treated mice w/o intragastrically administration of E. coli (Fig. 1b, upper left panel). The results showed that bigger primary tumors and more liver metastatic foci were detected in the E. coli group than that in the control group with statistical significance (Fig. 1b, upper right panel and lower panel). Immunohistochemistry and immunofluorescence assays indicated that intestinal dysbiosis brought by the excessive administration of $E$. coli dramatically increased the releasing of LPS in tumor microenvironment (Fig. 1c). Twenty-six differentially overexpressed genes in metastatic CRC tissues were identified through analyzing our previously published highthroughput microarray dataset of eight pairs of nonmetastatic and metastatic human CRC tissues (NCBI/ GEO/GSE113296; $n=8$, Fig. 1d). Their response to LPS was detected by quantitative PCR in two different CRC cell lines. Although CTSK, RCor2, and C9orf116 were significantly overexpressed in SW480 cells in response to LPS while CTSK and CDH15 were significantly overexpressed in RKO cells, CTSK was the only gene upregulated in both cell lines (Fig. 1e). The IHC staining of two representative cases of nmCRC and mCRC tissues confirmed a positive correlation between overexpression of CTSK and LPS releasing in the stroma $(P=0.022$; Fig. 1f). LPS could 

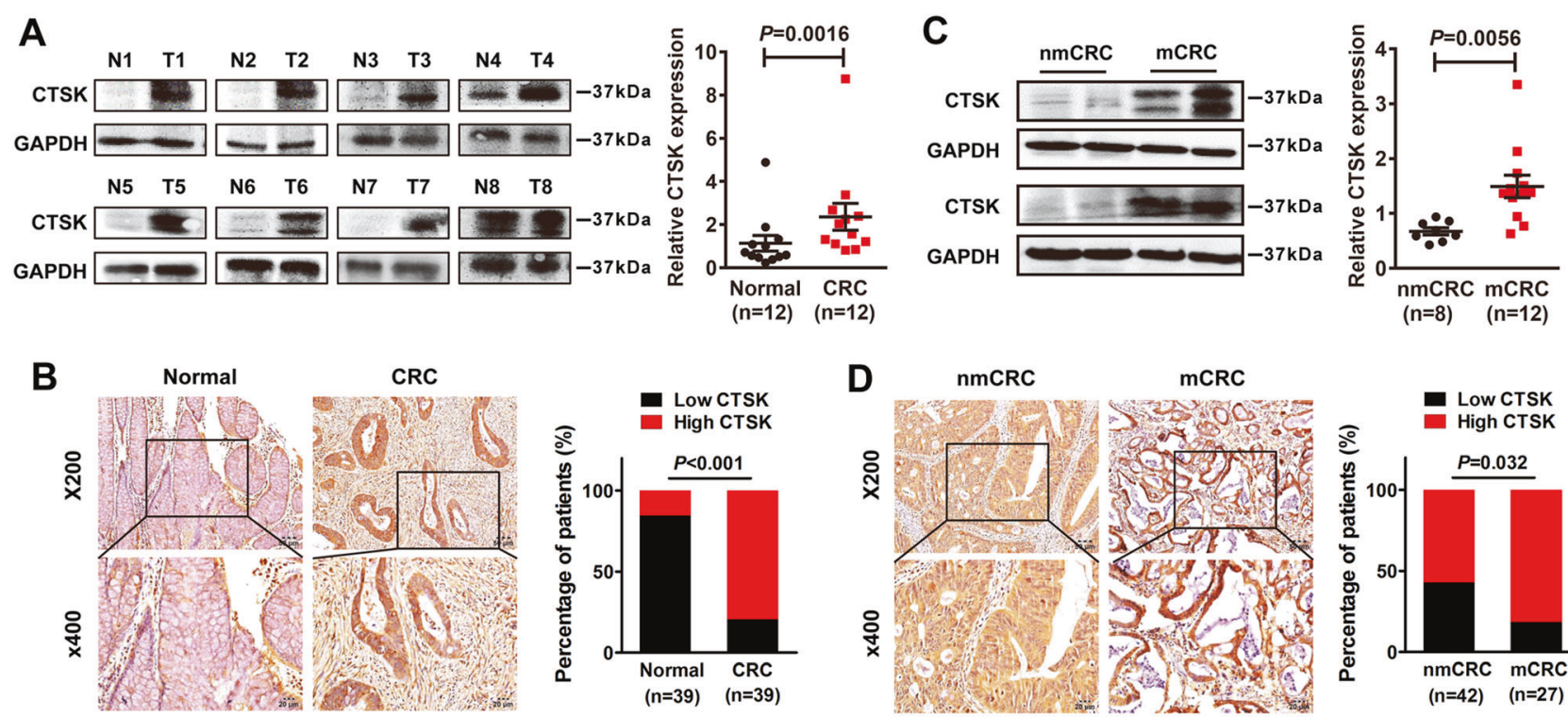

D
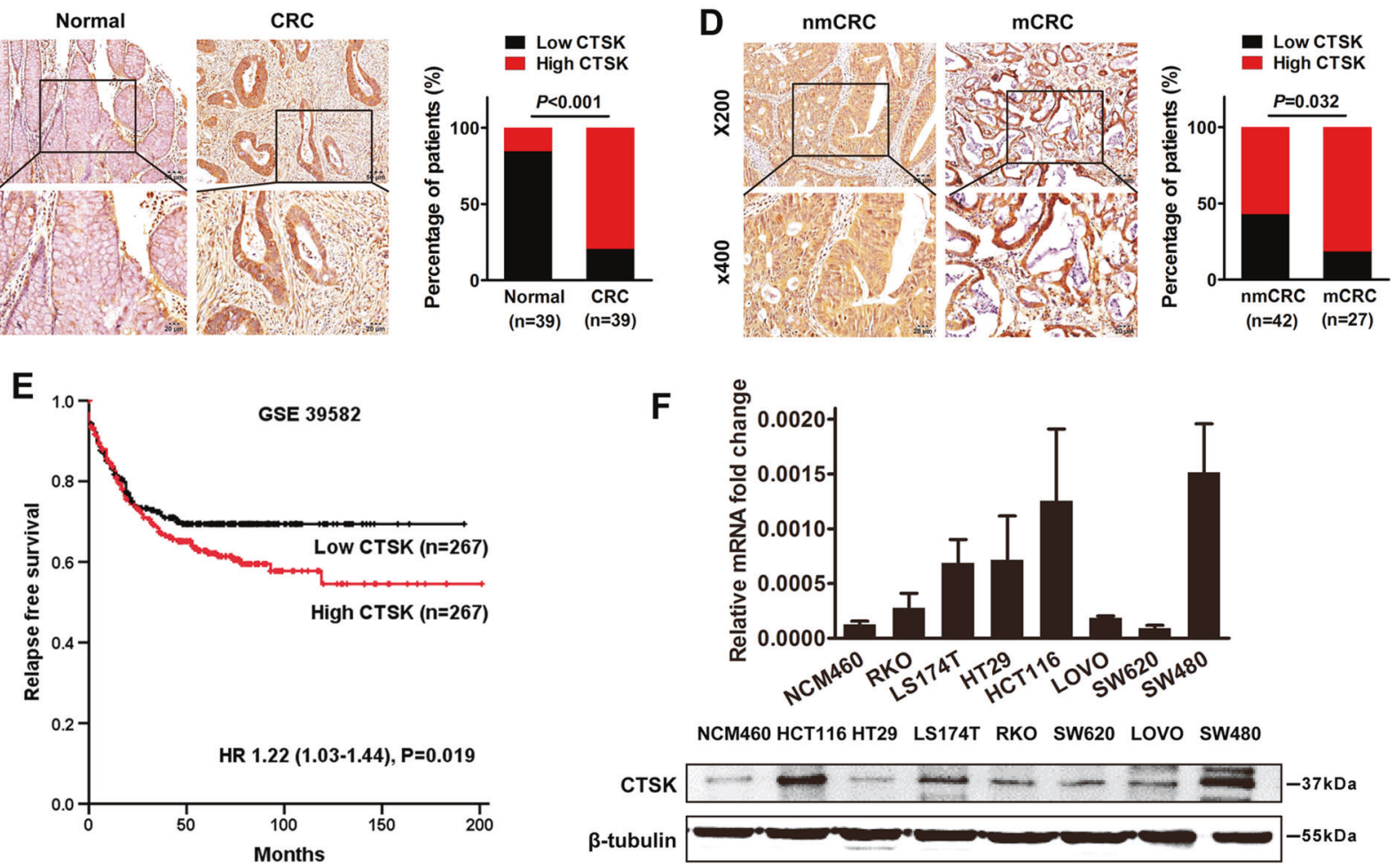

Fig. 2 Overexpression of CTSK is associated with advanced progression and poor prognosis of colorectal cancer. a Western blot analysis of CTSK in colorectal cancer (CRC) tissues (T) and adjacent non-tumor tissues $(\mathrm{N})$. The scatter chart in the right shows the relative CTSK expression in normal and CRC tissues (normalized to GAPDH, $P=0.0016$ ). b Representative images of immunohistochemistry (IHC) staining analysis of CTSK expression in CRC tissues and adjacent non-tumor tissues. The bar chart in the right represents the percentage of high and low CTSK expression cases in normal and CRC tissues. $\mathbf{c}$ Western blot analysis of CTSK in non-metastatic (nmCRC) and

metastatic (mCRC) colon cancer tissues. The scatter chart in the right shows the relative CTSK expression in $\mathrm{nmCRC}$ and $\mathrm{mCRC}$ tissues (normalized to GAPDH, $P=0.0056$ ). d IHC analysis of CTSK expression in non-metastatic and metastatic CRC tissues. The bar chart in the right represents the percentage of high and low CTSK expression cases in nmCRC and mCRC tissues. e Kaplan-Meier survival curves and univariate analyses (log-rank) for CRC patients with distinct expression levels of CTSK. f Western blot analysis was used to detect the expression of CTSK in CRC cell lines

increase the expression of CTSK protein in RKO and SW480 cells, but not in normal human colon epithelial FHC cells (Fig. 1g, upper panel). Similar results were obtained in those cells co-cultured with E. coli (Fig. 1g, lower panel).

\section{Overexpression of CTSK is associated with advanced progression and poor prognosis of colorectal cancer}

We analyzed the expression of CTSK protein in 12 CRC tissues. As shown in Fig. 2a, overexpression of CTSK was observed in eight out of 12 CRC tissues compared with matched non-tumor tissues $(P=0.0016)$. IHC results further demonstrated that CTSK was significantly upregulated in 79\% (31/39) of CRC tissues examined compared to that in $15 \%(6 / 39)$ of the adjacent normal tissues (Fig. $2 b, P<$ $0.001)$.

Moreover, western blot analysis demonstrated that CTSK protein is overexpressed in mCRC $(n=12)$ tissues compared with that of nmCRC tissues $(n=8)(P=$ 0.0056 , Fig. 2c). To evaluate the clinical significances of CTSK, IHC assay was further performed in $42 \mathrm{nmCRC}$ tissues and $27 \mathrm{mCRC}$ tissues. CTSK was overexpressed in 
A
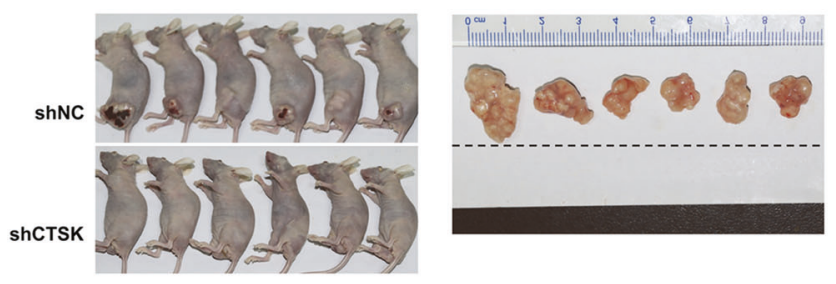

C

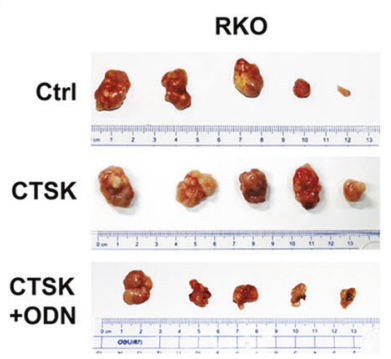

B

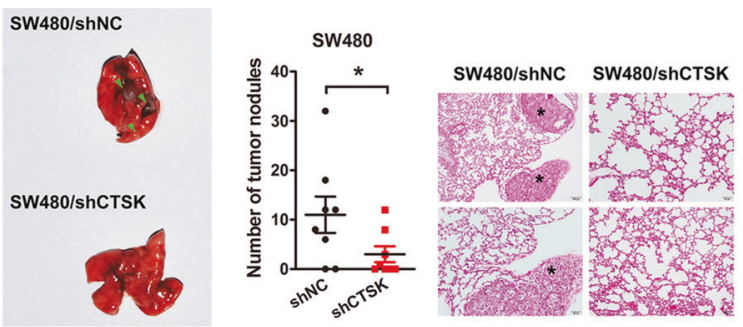

D

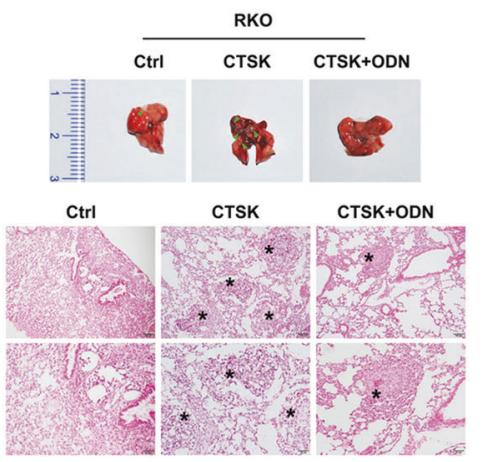

E

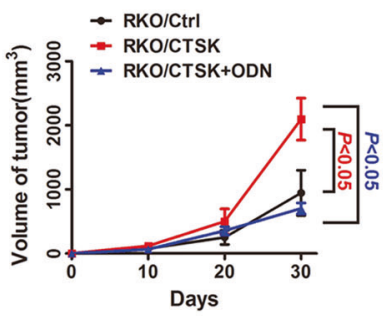

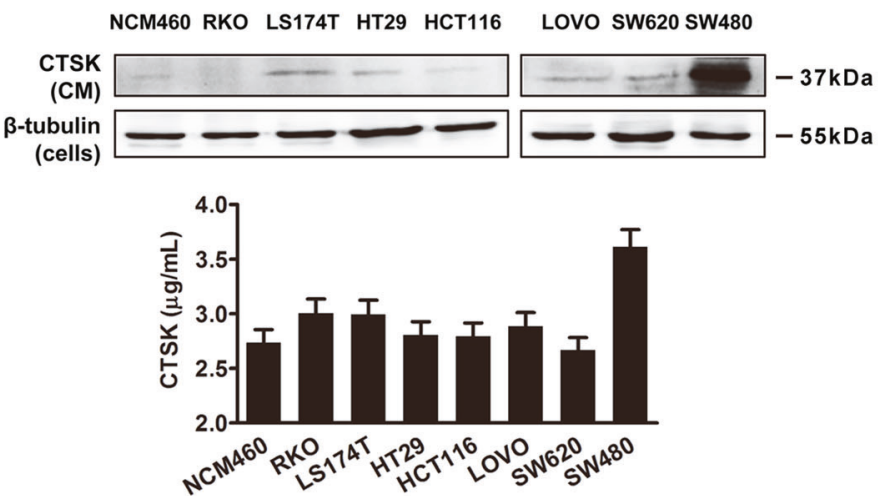
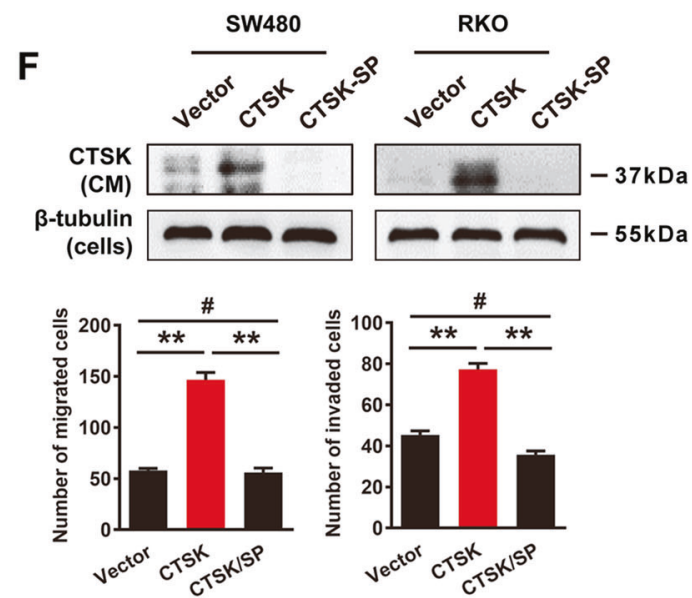

Fig. 3 Exogenous CTSK contributes to aggressive phenotypes of colorectal cancer (CRC) cells both in vitro and in vivo. a SW480 tumor cells were injected subcutaneously into the back of nude mice to evaluate tumor growth. Representative figure of tumors formed is shown. b The shNC and shCTSK-SW480 cells were injected into nude mice through the tail vein to evaluate the lung homing potential of cells. The number of metastatic lung nodules in individual mice was counted under the microscope. The magnification areas indicated metastatic nodes in the lung. The asterisk $\left(^{*}\right)$ indicates $P<0.05$. c The CTL and CTSK overexpression RKO cells were injected subcutaneously into the back of nude mice to evaluate tumor growth with

$38 \%(16 / 42)$ of nmCRC tissues and a much higher rate was observed in $81 \%(22 / 27)$ of mCRC samples $(P=$ 0.032 , Fig. 2d). Kaplan-Meier survival analysis of a previously published CRC dataset (NCBI/GEO/GSE $39582, n=534$ ) revealed that CTSK expression is closely correlated with patients' survival rate and life expectancy. Patients with low CTSK expression have a significantly better prognosis (Fig. 2e). or without ODN (3.606 $\mathrm{mg} \mathrm{kg}^{-1}$ per week) treatment. Representative figure of subcutaneous tumors are shown. d The Ctrl and CTSK overexpression RKO cells were injected into nude mice through the tail vein to evaluate the lung homing potential of cells with or without ODN (3.606 $\mathrm{mg} \mathrm{kg}^{-1}$ per week) treatment. The number of metastatic lung nodules in individual mice was counted under the microscope. The magnification areas indicated metastatic nodes in the lung. The asterisk (*) indicates $P<0.05$. e Western blot analysis shows the expression of CTSK in conditioned media (CM) of CRC cell lines. $\mathbf{f}$ Western blot analysis was used to detect the secretion of CTSK in conditioned media (CM) of CRC cell lines

\section{Exogenous CTSK contributes to aggressive phenotypes of CRC cells both in vitro and in vivo}

Real-time PCR and western blot assays were used to measure the expression of CTSK in CRC cell lines. Compared with NCM460, CTSK in overexpressed in CRC cells at either mRNA or protein level (Fig. 2f). To further investigate the effects of CTSK on the biological behaviors of 
A

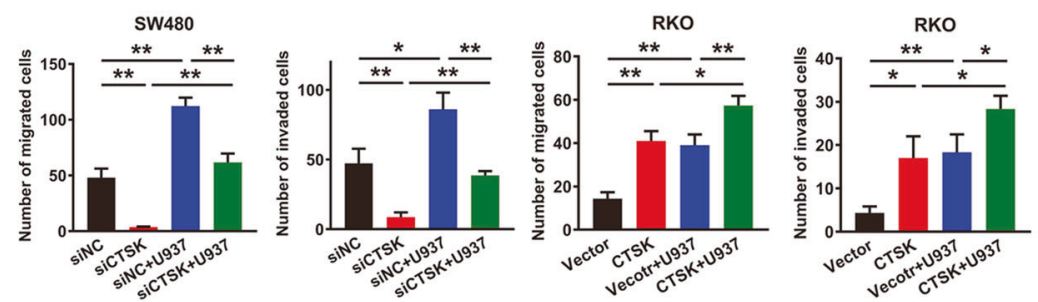

B
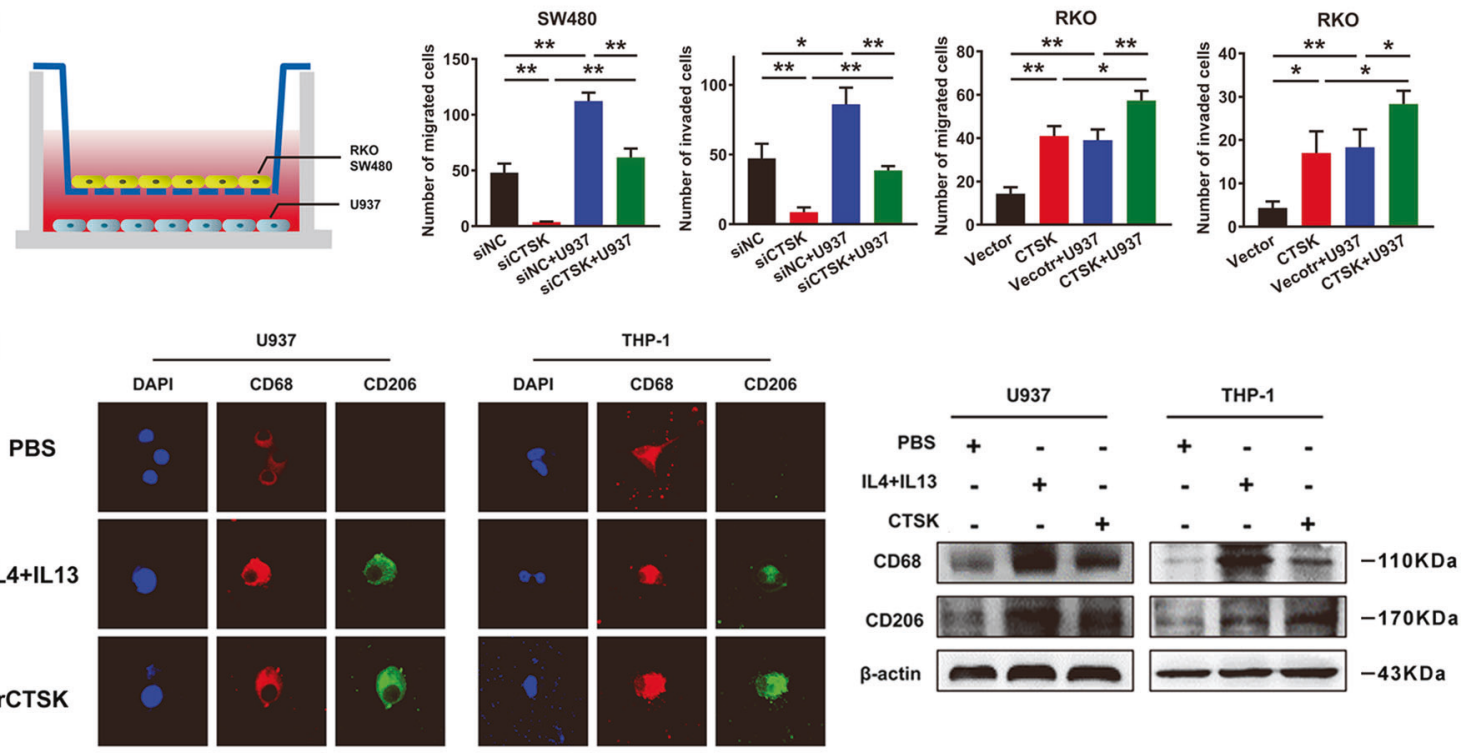

rCTSK

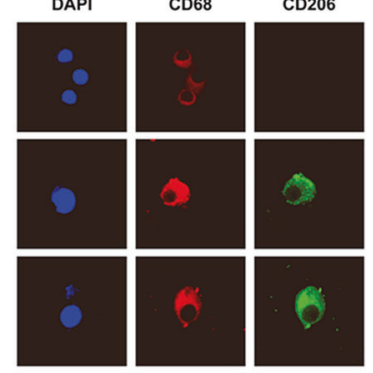

C

D
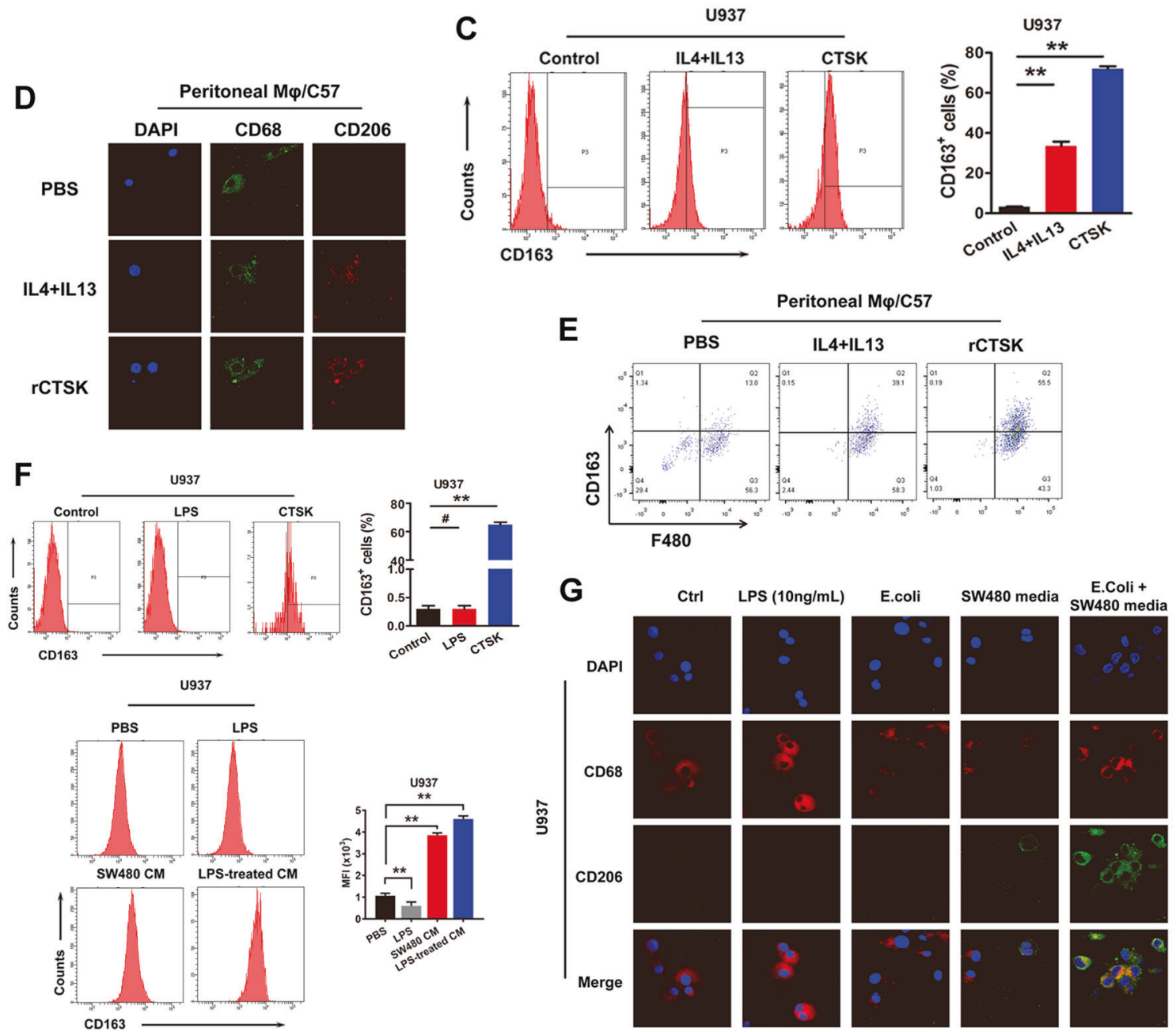

CRC cells, we successfully constructed the CTSK overexpressed lentivirus (LV-CTSK), CTSK silencing vector (shCTSK), and synthesized two CTSK small interfering
RNAs (siRNAs; siCTSK1 and siCTSK2). Endogenous CTSK lowly expressed RKO cells and CTSK highly expressed SW480 cells were chosen to overexpress or 
Fig. 4 Tumor-secreted CTSK contributes to colorectal cancer (CRC) cell migration via inducing the M2 polarization of macrophages. a Schematic diagram shows a co-culture system of macrophage-like U937 cells and CRC cells. Bar charts shows the invasion and migration ability of CRC cells with or without the existence of macrophagelike U937 cells. The asterisk $(*)$ indicates $P<0.05$. The double asterisk $(* *)$ indicates $P<0.01$. b Left panel: IF assay was used to visualize the increase of CD206 in macrophage-like U937 and THP-1 cells treated with $\operatorname{rCTSK}(0.1 \mu \mathrm{g} / \mathrm{ml})$ or IL $4 / \mathrm{IL} 13(0.1 \mu \mathrm{g} / \mathrm{ml})$. Right panel: Western blot analysis were used to detect the expression of CD68 and CD206 in macrophage-like U937 and THP-1 cells treated with recombinant protein CTSK $(0.1 \mu \mathrm{g} / \mathrm{ml})$ or IL $4 / \mathrm{IL} 13(0.1 \mu \mathrm{g} / \mathrm{ml})$. c Flow cytometry shows the number of $\mathrm{CD} 163^{+} \mathrm{U} 937$ cells induced by CTSK and IL4/IL13. d IF assay was used to visualize the expression of CD206 in peritoneal macrophages treated with $\operatorname{rCTSK}(0.1 \mu \mathrm{g} / \mathrm{ml})$ or IL4/IL13 $(0.1 \mu \mathrm{g} / \mathrm{ml})$. e Flow cytometry analysis shows the effects of rCTSK and IL4/IL13 on the M2 polarization of peritoneal macrophages. f Upper panel: Flow cytometry analysis shows the effect of lipopolysaccharide (LPS) and CTSK on the polarization of tumorassociated macrophages (TAMs). Lower panel: Flow cytometry analysis shows that LPS could only stimulate M2 Polarization of TAMs with the existence of CRC cells. g IF assay was used to visualize the increase of CD206 in macrophage-like U937 cells treated with LPS or the conditioned media of SW480 cells with or without the existence of E. coli

silence CTSK, respectively. Transfection efficiency was confirmed by western blot analysis (Fig. S1A). Exogenous overexpression of CTSK dramatically enhanced the migration and motility ability of RKO cells in 3D invasive, transwell, and wound healing assays (Fig. S1B-D). Meanwhile, silencing of CTSK inhibited the migration and invasion of SW480 cells. Although the in vitro CCK8 and clone formation assays did not support a stimulatory of CTSK in CRC cell proliferation (Fig. S2), silencing of CTSK did dramatically inhibit the growth of subcutaneous tumors (4.2 $\pm 0.5 \mathrm{~g}$ vs. $0 \mathrm{~g}, P<0.05$; Fig. 3a). Moreover, no significant lung metastatic focus was found in the shCTSK group, while the average number of pulmonary metastatic foci in the shCtrl group was 2.9 (Fig. 3b). Intragastric administration of ODN, a CTSK-specific inhibitor, reversed the CTSK promoted subcutaneous tumor growth and pulmonary metastatic foci formation (Fig. 3c, d).

\section{CRC-secreted CTSK contributes to migration and motility ability of CRC cells}

As a secretory protein, the concentration of CTSK in the culture medium of NCM460 cells and seven CRC cells was detected by ELISA assay (Fig. 3e). Western blot assay indicated an increased CTSK secretion in response to LPS in RKO and SW480 cells (Fig. S3). CTSK overexpressed plasmid without signal peptide (CTSK-SP) was constructed to address the effect of CRC-secreted CTSK on the migration and motility of CRC cells. Introduction of CTSKSP significantly reduced the CRC-secreted CTSK, as well as decreased the migration and invasion of CRC cells, compared with the full-length CTSK overexpression group, suggesting an indispensable role of tumor-secreted CTSK in CRC metastasis (Fig. 3f). Similar results were obtained when Odanacatib (ODN) was administrated to the culture medium (Fig. S4) or endogenous CTSK was silenced by siRNA (Fig. S5).

\section{CRC-secreted CTSK stimulates CRC metastasis via inducing the $\mathrm{M} 2$ polarization of macrophages}

To validate whether CTSK functions as a potent CRCsecreted chemoattractant, we constructed a novel co-culture system using CRC cells and macrophages (Fig. 4a). PMAprimed macrophage-like U937 cells were applied in the bottom layer, while SW480 or RKO CRC cells was placed in the upper layer. CTSK silencing SW480 cells showed an increased ability of migration and invasion with the presence of macrophage-like U937 cells compared with that without primed U937 cells. Consistently, the CTSK stable overexpressing RKO cells displayed a significant increase in the ability of migration and invasion with the presence of U937-derived macrophage-like cells.

TAMs show anti-(M1) or protumor (M2) functions depending on the cytokine milieu of the tumor microenvironment. We applied M2-specific markers (CD163 and CD206) to address whether CRC-secreted CTSK contributes to CRC metastasis through stimulating M2 polarization of TAMs. Similar to the effects of IL4/IL13, recombinant CTSK (rCTSK) significantly increased the expression of CD206 and CD68 in macrophage-like U937 cells and THP-1 cells detected by immunofluorescence staining (Fig. 4b, left panel). Similar conclusion was drawn by western blot assay through detecting the expression of CD206 and CD68 proteins (Fig. 4b, right panel). Moreover, the number of CD163-positive macrophage-like U937 cells detected by flow cytometry analysis was dramatically increased after the administration of CTSK (Fig. 4c). In consistent, rCTSK could also increase the expression and the number of CD206 and CD163-positive cells in primary peritoneal macrophages detected by immunofluorescence and flow cytometry assays (Fig. 4d, e).

In the primary tumors developed by orthotopic implantation of MC38 cells in antibiotic-treated mice, excessive LPS induced by intragastrically administration of $E$. coli remarkably stimulated the expression of CTSK, as well as the number of CD68 and CD163 -positive macrophages in tumor stroma (Fig. S6). Interestingly, a stimulatory effect of LPS on M2 polarization of TAMs could only be detected with the existence of CRC cells (Fig. 4f). Similarly, immunofluorescence staining indicated that only the $E$. coli pretreated SW480 CM could significantly increased the expression of CD206 and CD68 in macrophage-like U937 cells, LPS or E. coli alone could not induce the M2 polarization of macrophages (Fig. 4g). 
A

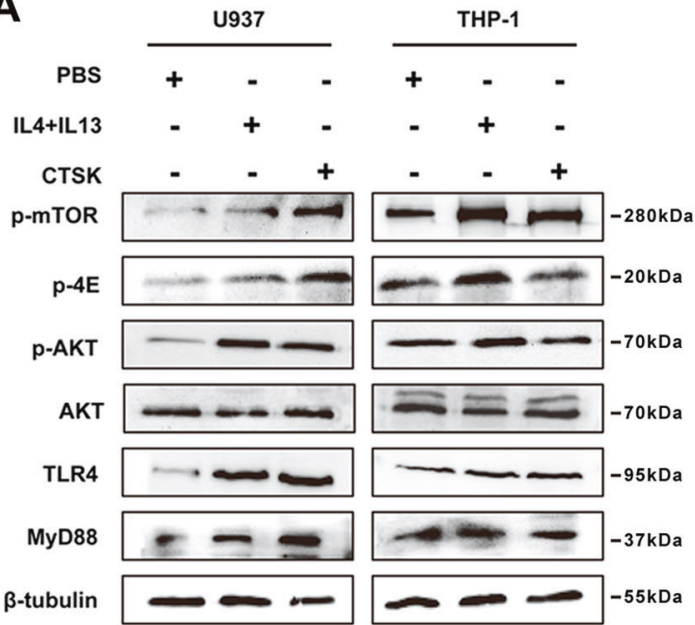

B

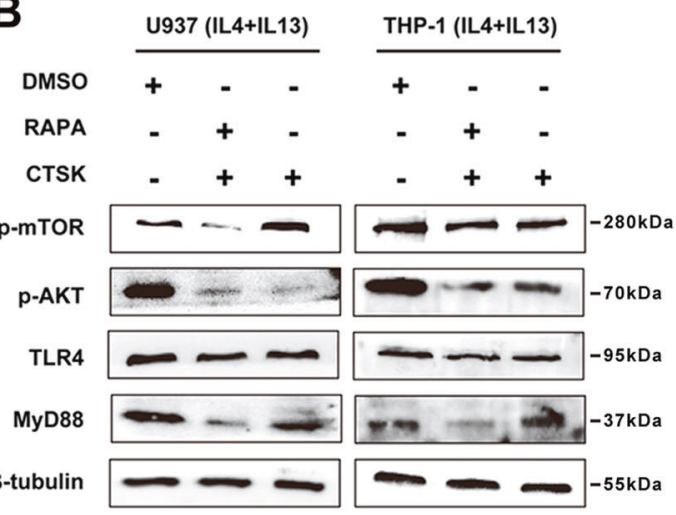

C
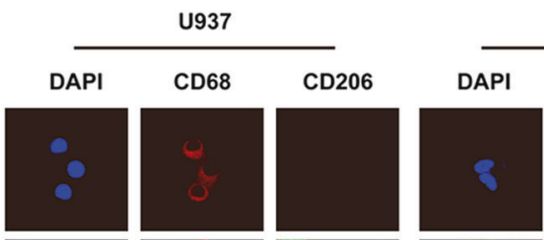

THP-1
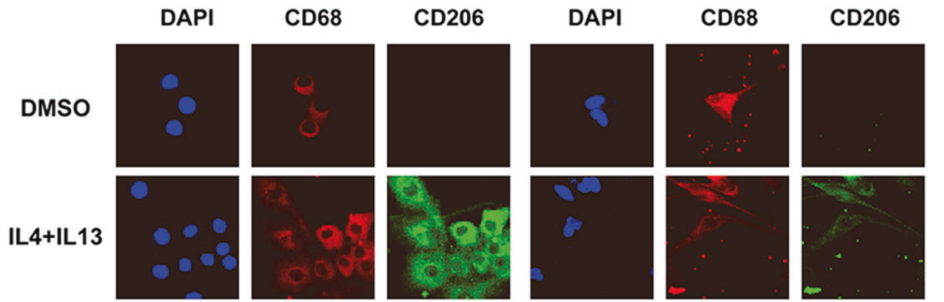

\section{D}

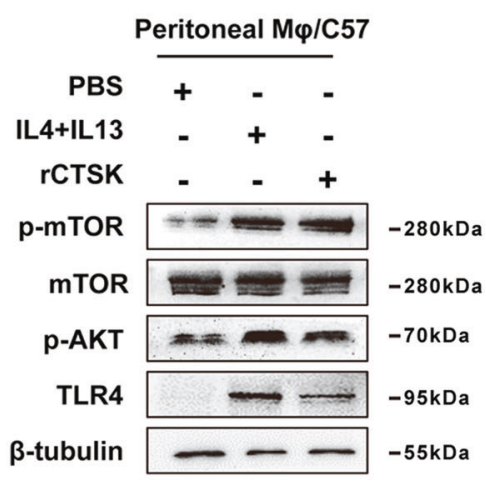

E
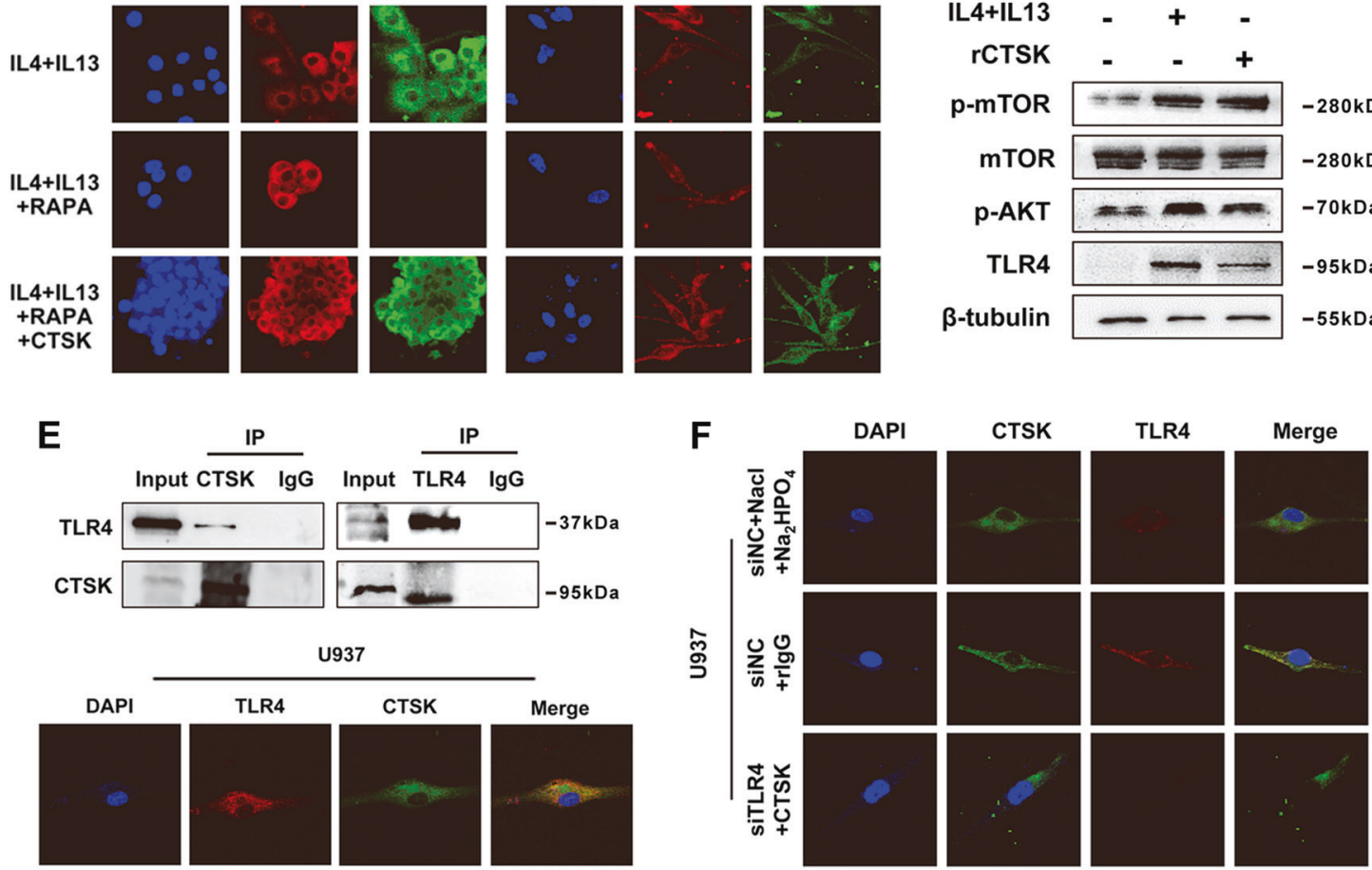

Fig. 5 Colorectal cancer (CRC)-secreted CTSK activates mTOR pathway via interaction with macrophage membrane receptor Toll-like receptor 4 (TLR4). a Western blot analysis detected the expression of TLR4/MyD88p-AKT/mTOR pathway members (phosphorylated AKT at Ser473, p-mTOR, p-4E, TLR4, MyD88) in macrophage-like cells w/o the treatment of rCTSK and IL4/IL13. b Western blot analysis of p-AKT/mTOR pathway members, TLR4 and MyD88 in M2 type macrophage-like cells with or without the treatment of rCTSK and Rapamycin (100 nmol). c IF assay analysis of the expression of CD68

and CD206 in IL4/IL13 induced M2 type macrophage-like cells w/o treatment of rCTSK and Rapamycin. d Western blot analysis detected the expression of TLR4/MyD88p-AKT/mTOR pathway members in peritoneal macrophages w/o the treatment of rCTSK and IL4/IL13. e Upper panel: Validation of endogenous interaction between CTSK and TLR4 in indicated cells. Lower panel: IF assay analysis of the colocalization of CTSK with TLR4 in macrophage-like cells. f IF assay shows the decreased co-localization of CTSK and TLR4 in macrophage-like cells after silencing TLR4 


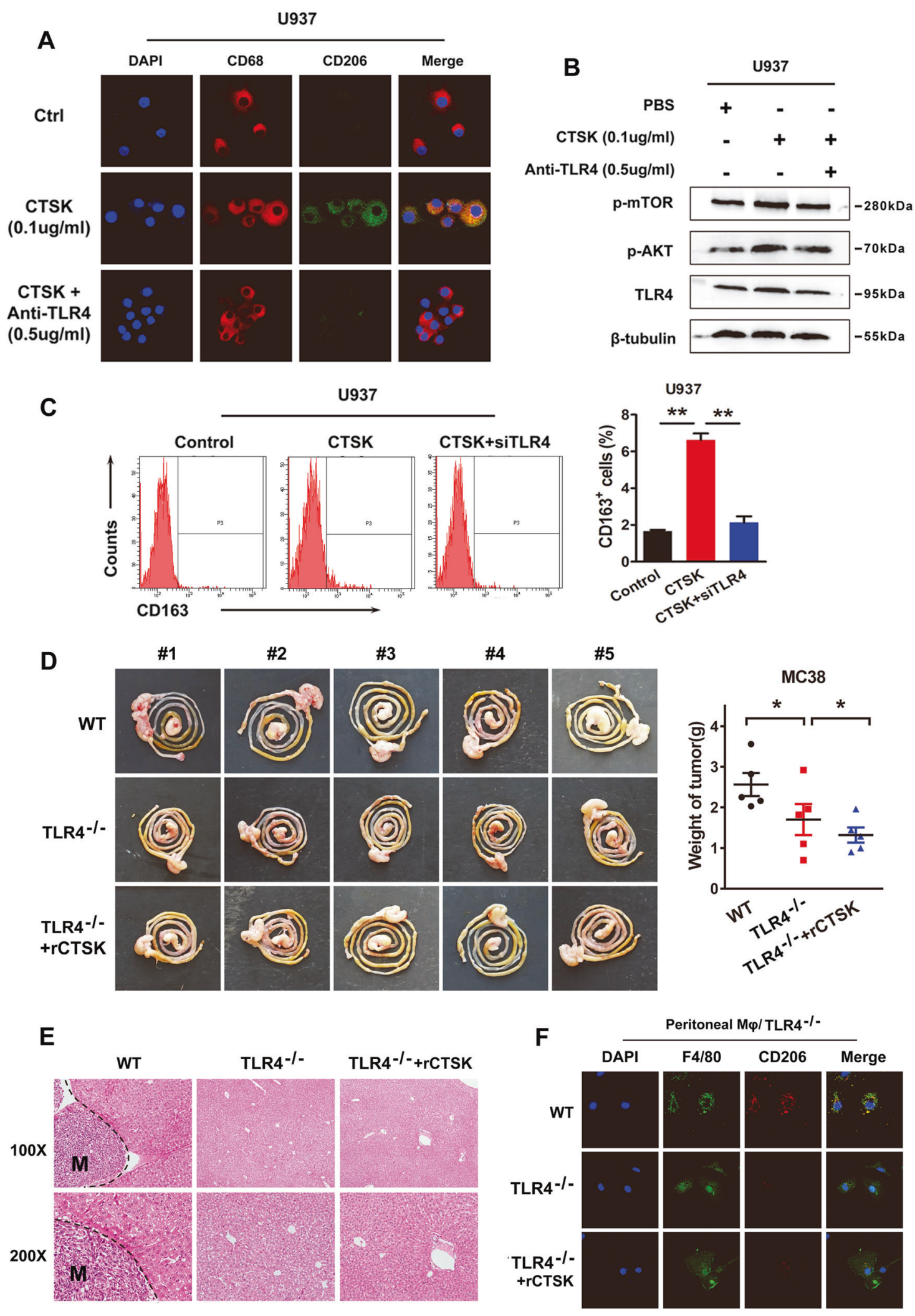


Fig. 6 TLR4 pathway is essential for CTSK-induced M2 polarization of macrophages and colorectal cancer (CRC) cell migration. a IF assay represents the decreased co-localization of CTSK and TLR4 in rCTSK activated macrophage-like cells after administration of TLR4 antibody. b Western blot analysis detected the expression of TLR4-AKT/mTOR pathway members in rCTSK stimulated U937 macrophage-like cells after the treatment of TLR4 antibody. c Flow cytometry analysis shows the number of CD163 in macrophage-like cells in indicated cells. d The primary tumor developed by implantation of MC38 cells in WT and TLR4 ${ }^{-1-}$ mice w/o intraperitoneal injection of rCTSK. Scatter diagram on the right represents the weight of tumors. e HE staining shows the hepatic metastasis foci in in WT and TLR $4^{-1-}$ mice w/o intraperitoneal injection of rCTSK. f IF assay analysis of the expression of F4/80 and CD206 in peritoneal macrophages larvaged from WT and TLR4 ${ }^{-1-}$ mice w/o treatment of rCTSK

\section{CRC-secreted CTSK activates mTOR pathway via interaction with macrophage membrane receptor Toll-like receptor 4 (TLR4)}

We explored possible oncogenic pathways involving in CTSK-mediated M2 polarization of TAMs. Western blot analysis showed that mTOR pathway, a key intracellular signaling system that drives cellular growth and survival, was activated in PMA-primed U937 and THP-1 cells after administration of IL4/IL13 or rCTSK (Fig. 5a). Rapamycin, the specific inhibitor of mTOR signaling, suppressed the IL4/IL13 activated expression of p-Akt and p-mTOR, whereas administration of rCTSK could reverse the rapamycin caused inhibition of mTOR signaling (Fig. 5b). Immunofluorescence staining of primed U937 and THP-1 cells also confirmed the involvement of mTOR signaling in CTSK-mediated M2 polarization of TAMs (Fig. 5c). Western blot results showed that administration of rCTSK also increased the expression of TLR4 and activated the PI3K/ AKT/mTOR signaling pathway in mouse peritoneal macrophage culture (Fig. 5d).

Previous reports suggested that CTSK might function through toll-like receptor family [22]. In our study, the activation of classic TLR4/Myd88 pathway was confirmed in response to rCTSK (Fig. 5a). Moreover, silencing of CTSK was also accompanied with a decreased expression of TLR4 in CRC cells (Fig. S7). Interaction between CTSK and TLR4 was further validated by coimmunoprecipitation (Co-IP) assay using protein extractions of CTSK highly expressed SW480 cells (Fig. 5e, upper panel). Immunofluorescence staining indicated a co-localization of CTSK and TLR4 in macrophage-like U937 cells (Fig. 5e, low panel), whereas silencing of TLR4 eliminated their co-localization (Fig. 5f). TLR4 antibody obviously decreased rCTSK induced expression of CD206 in U937 cells (Fig. 6a) and inhibited the activation of CTSK-induced mTOR pathway (Fig. 6b). Consistently, the number of CD163-positive cells stimulated by rCTSK was dramatically reduced in
TLR4 silencing macrophage-like U937 cells (Fig. 6c). To further validate the involvement of the TLR4-mTOR pathway in CTSK-induced M2 polarization of TAMs, we implanted MC 38 cells to the cecum of TLR $4^{-l-}$ mice. The results showed that both the tumor volume and the number of hepatic metastasis foci in TLR $4^{-/}$mice were significantly reduced compared with WT mice. CTSK could not stimulate the growth and metastasis of orthotopically implanted tumors in TLR $4^{-1-}$ mice (Fig. 6d, e). Moreover, immunofluorescence (Fig. 6f) and flow cytometry (Fig. S8) assays showed that exogenous CTSK could not induce the M2 polarization of macrophages in $\mathrm{TLR}^{-1-}$ mice. To sum up, interaction of TLR4 with CTSK is essential for the polarization of M2 macrophages.

\section{CTSK-induced cytokines secretion by M2 TAMs promotes CRC cells migration via NF-KB signaling pathway}

Recent studies indicated that the M2 TAMs play a supportive role in tumor progression by secreting cell growth cytokines and survival factors [23]. Hence, we applied Human Cytokine Antibody Array to detect the changes of cytokines secretion in the culture medium of PMA-primed U937 cells before and after rCTSK treatment. As shown in Fig. 7a, administration of rCTSK could significantly increase the secretion of chemokines, such as IL4, IL10, and IL17. More importantly, overexpression or silencing CTSK could promote or inhibit the expression of membrane cytokine receptors in CRC cells correspondingly, indicating that CTSK recruited M2 TAMs might promote CRC progression through increased secretion of cytokines (Fig. 7b). Applying those cytokines to SW480 CRC cells also significantly promoted their migration and motility abilities (Fig. 7c).

Previous reports suggested that cytokines, such as IL10 and IL17, are key regulators for NF- $\mathrm{kB}$ (P65) pathway in mediating cell migration [23]. Consistently, immunofluorescence staining showed that recombinant IL10 and IL17 could promote the nuclear translocation of phos-

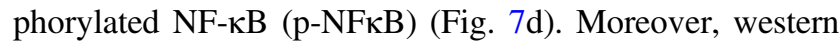
blot analysis also indicated a stimulatory role of those cytokines on the nuclear translocation of $\mathrm{p} 65$ and cytoplasm translocation of IкB kinase alpha (IKK- $\alpha$ ). Meanwhile, the

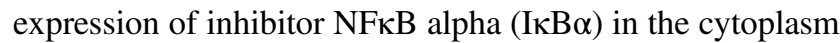
was relatively attenuated (Fig. 7e). BAY11-7082, the special inhibitor of NF- $\mathrm{KB}$ pathway, was applied to elucidate involvement of NF- $\mathrm{KB}$ signaling pathway in cytokinesmediated migration and invasion of CRC cells. Administration of BAY11-7082 could dramatically suppress cytokines (IL10 and IL17) stimulated invasion of CRC cells (Fig. 7f). To further validate whether CRC-secreted CTSK 
A

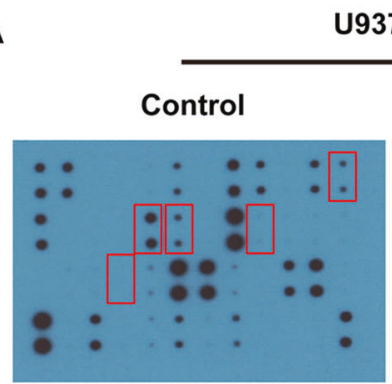

937

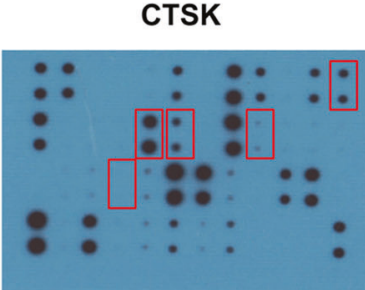

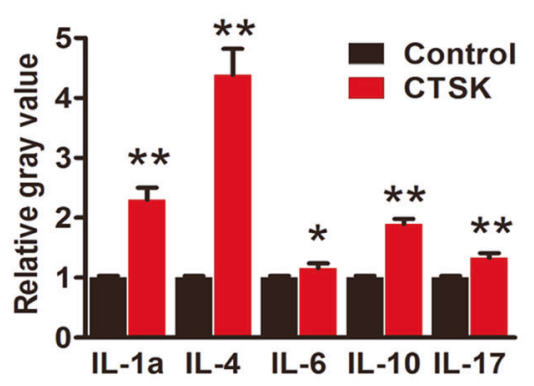

SW480
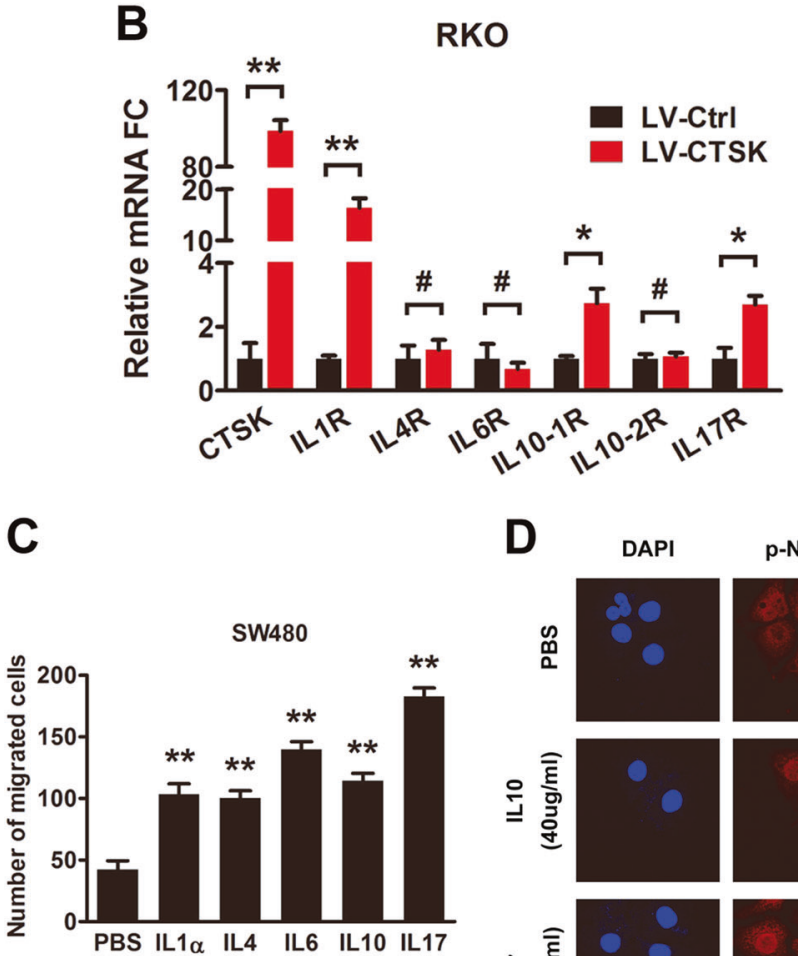

F

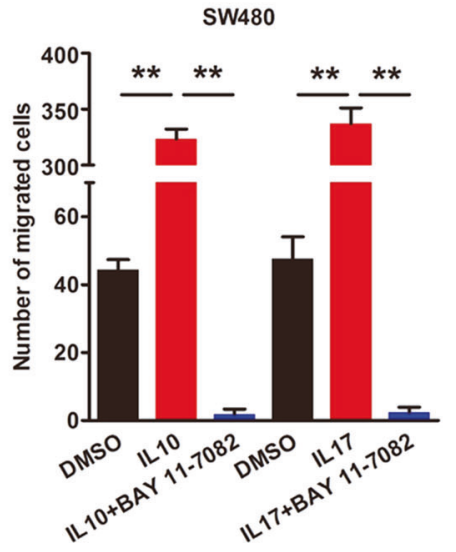

D DAPI

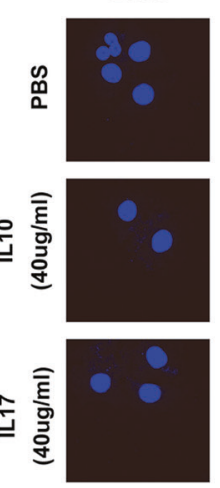

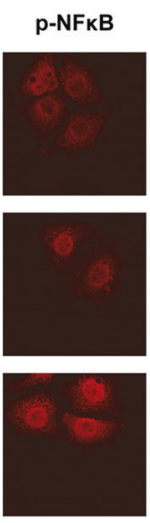

G

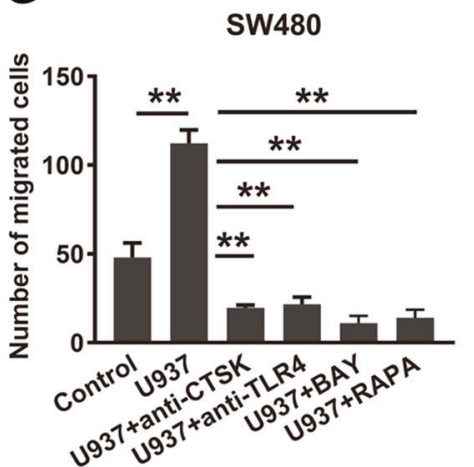

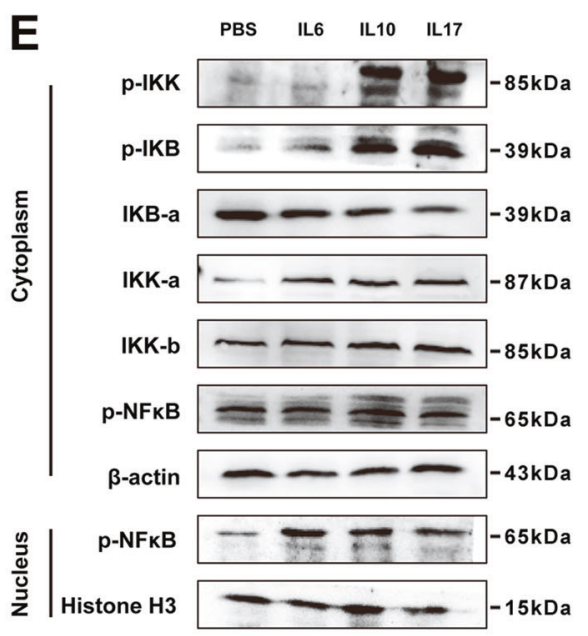

SW480

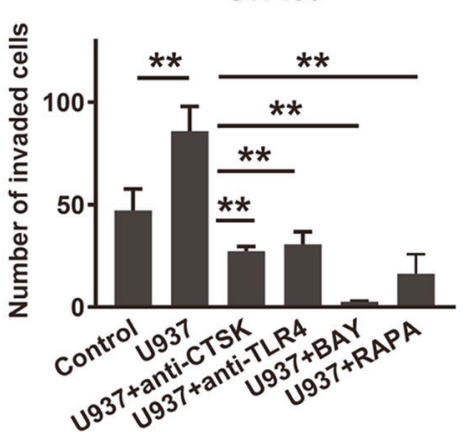

increases CRC cell migration and invasion through M2 TAMs-mediated TLR4-mTOR-NFKB pathway, CTSK, and TLR4 antibodies, as well as inhibitors of mTOR and NFKB pathways were applied. As expected, blockage of the pathway at each point could abolish almost all the downstream effects (Fig. 7g). 
Fig. 7 Colorectal cancer (CRC)-secreted CTSK-induced cytokines secretion promotes CRC cells invasion and metastasis via NF- $\kappa B$ signaling pathway. a Human Cytokine Antibody Array was applied to detect the changes of inflammatory factors in conditioned media (CM) of macrophage-like cells treated with recombinant protein CTSK. The asterisk (*) indicates $P<0.05$. The double asterisk (**) indicates $P<$ 0.01. b Real-time PCR analysis shows the expression of cytokine receptors in CTSK overexpression or silencing CRC cells. c The number of invading cells in transwell assay with the treatment of different cytokines was counted under a microscope in five randomly selected fields. d IF assay was used to visualize the nucleus translocation of p-NFkb with the treatment of IL10 or IL17. e Western blot analysis of NFkb pathway members in nuclear and cytoplasm of CRC cells treated with IL10 or IL17. f The bar chart shows the number of invaded cells in transwell assay with or without the treatment of BAY11-7082. g The bar chart shows the invasion and migration of CRC cells in the co-culture system with the treatment of CTSK antibody, TLR4 antibody, BAY11-7082, and Rapamycin

\section{Overexpression of CTSK is correlated with high abundance of M2 TAMs in CRC tissues}

Immunofluorescence staining demonstrated that high CTSK expression in CRC tissues is always accompanied with high abundance of M2 TAMs in tumor stroma (Fig. 8a). Similar result was obtained by IHC staining of human CRC specimens. Overexpression of CTSK is positively correlated with the metastatic status of CRC and the abundance of M2 TAMs (CD163 positive) in the stroma (Fig. 8b, left panel). Moreover, the number of $\mathrm{CD}_{163^{+}}$cells counted under high power field (HPF) in the stroma of high CTSK specimens is nearly twice of that in low CTSK specimens (Fig. 8b, right panel, $P<0.01)$.

\section{Discussion}

$\mathrm{CRC}$ is frequently associated with alterations of intestinal microbiota composition and structure [24]. The imbalance of intestinal microbiota alternates the construction of inflammatory microenvironment and promotes CRC initiation and progression [25]. Although numerous studies have demonstrated the inflammatory response of intestinal dysbiosis on CRC progression, the underlying mechanism remains undefined.

Altered gut microbiota is usually accompanied with increased levels of LPS, a specific antigen of bacteria [26]. Here, an increased LPS was detected in mCRC tissues, indicating a stimulatory role of intestinal dysbiosis on CRC metastasis. This was further confirmed in the MC38 cells implanted antibiotic-treated mice w/o intragastrically administration of E. coli. Bigger primary tumors and more hepatic metastasis foci were developed in the E. coli group, which is accompanied with excessive LPS secretion. In this study, CTSK was identified as a metastasis-related gene for the first time, and it could be upregulated by LPS. Consistent with the stimulatory role of CTSK on aggressive phenotypes of other cancer types [12, 27, 28], CTSK stimulated the migration and invasion of CRC cells. Although CTSK did not increase CRC cell proliferation in vitro, no subcutaneous tumor was formed in the CTSK silencing group, suggesting a vital role of tumor microenvironment in CTSK-mediated CRC progression. Further analysis showed that only CRC-secreted CTSK is essential in promoting the aggressive phenotype of CRC cells. In pulmonary metastasis model, administration of Odanacatib can significantly suppress cancer cell metastasis, suggesting that CTSK inhibitors might be used as a novel therapeutic strategy for preventing tumor metastasis.

Apart from the well-known function of CTSK in extracellular matrix degradation and remodeling, it could also participate in the activation of immune cells, especially macrophages [29]. The expression of macrophageassociated factor CCL2 was significantly reduced in cathepsin K-deficient (ctsk-/-) mice, indicating a stimulatory role of CTSK in macrophages recruitment and tumor metastasis [28]. In this study, administration of PMAprimed macrophage-like U937 cells dramatically increased the migration and motility ability of CRC cells, whereas silencing of CTSK would counteract the aggressive phenotype of CRC cells. Hence, tumor-secreted CTSK might mediate the imbalance of microbiota to activate macrophages in microenvironment, which in turn stimulate the aggressive phenotype of CRC cells.

In tumor microenvironment, tumor-secreted chemokines and growth factors could induce monocytes recruitment and differentiation to macrophage. With the influence of those cytokines signals, TAMs undergo polarization into M1 and M2 phenotypes. M1 macrophages express a series of proinflammatory cytokines and chemokines to inhibit tumor progression, whereas M2 macrophages express antiinflammatory cytokines, chemokines, and proteases to promote tumor progression and immunosuppression. The immune-related factors including IL10, TGF-GF, EGF, VEGF, and MMPs, released by the M2 macrophages are regarded as the key component of CRC metastasis [30]. Moreover, the high density of M2 macrophages is related to shorter survival time and a high risk of recurrence in a clinical study in $120 \mathrm{CRC}$ patients [31, 32]. Therefore, we hypothesized that CRC-secreted CTSK promoted the invasion and migration of CRC by inducing the $\mathrm{M} 2$ polarization of macrophages. As expected, the stimulatory role of rCTSK on the recruitment of CD163 and CD206-positive M2 macrophages was detected. Our data also indicated that CTSK recruited M2 macrophages via an mTOR-dependent pathway, which is also important for macrophage activation [33]. However, LPS in non-tumor inflammatory environment without the existence of CRC cells could not stimulate 
A

Case 1 (High CTSK)

Case 2 (Low CTSK)
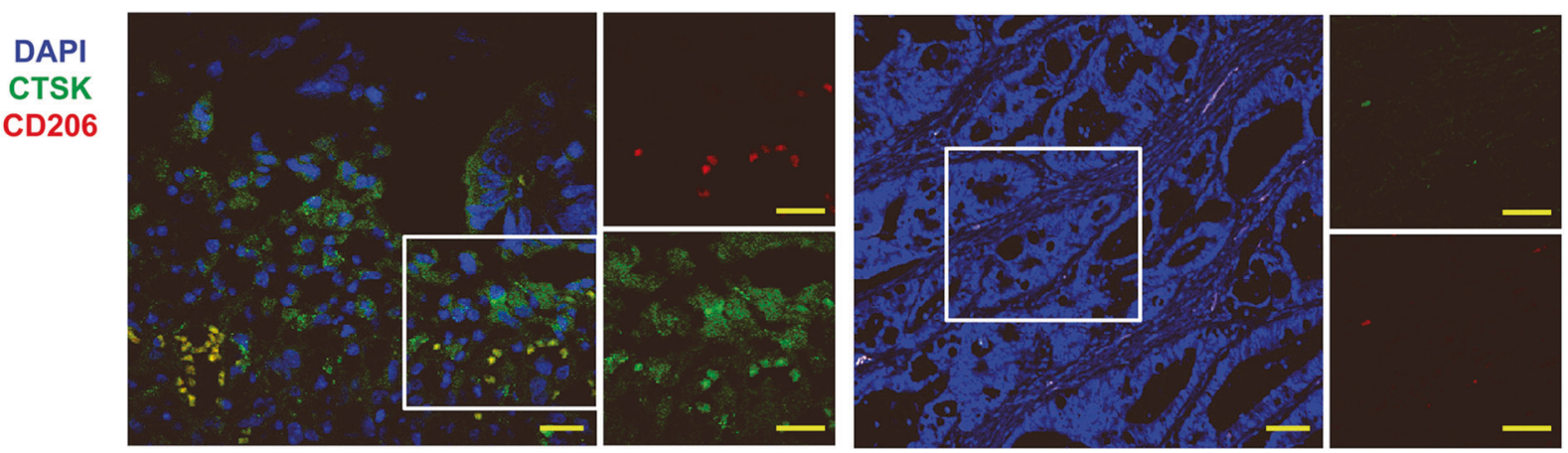

B nmCRC (Low CTSK)
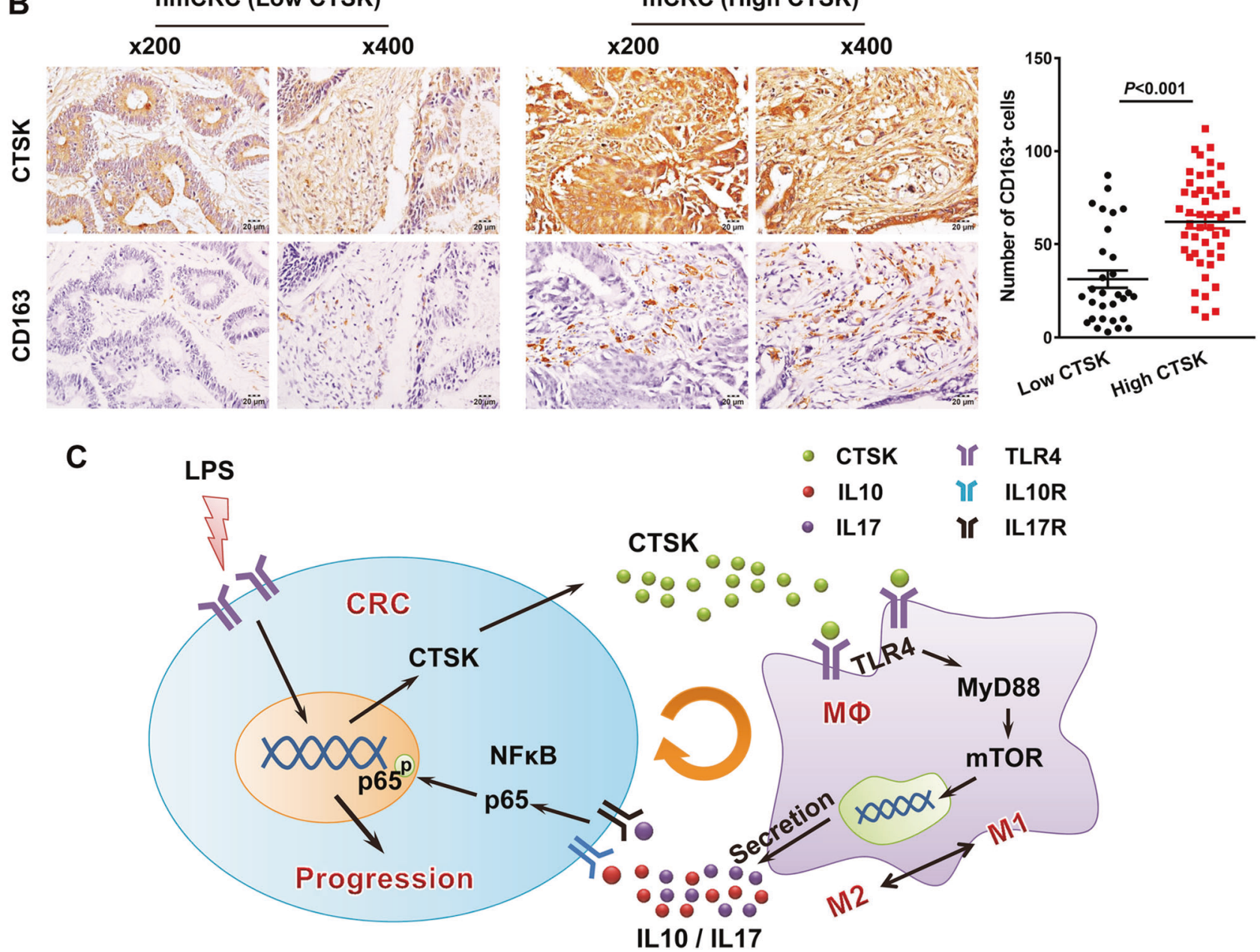

Fig. 8 Overexpression of CTSK is correlated with high abundance of M2 tumor-associated macrophages (TAMs) in colorectal cancer (CRC) tissues. a IF assay analysis of the correlation between CD206 and CTSK in colon cancer tissues. b Immunohistochemistry (IHC)

analysis of the correlation between CD163 and CTSK in colon cancer tissues. The asterisk $(*)$ indicates $P<0.05$. The double asterisk $(* *)$ indicates $P<0.01$. c The sketch map of the regulation and mechanism of CTSK-mediated CRC metastasis

M2 macrophages recruitment, which further confirmed the vital role of tumor-secreted CTSK in LPS-mediated CRC progression.

As LPS is a natural activator of toll-like receptors [34], the interaction between CTSK and toll-like receptors was studied. Toll-like receptors are a group of protein recognition receptors that recognize microorganisms and cause immune response, which are also demonstrated involving in in tumorigenesis [35]. Toll-like receptors are either upregulated or downregulated in response to CTSK in 
osteoarthritis [36], rheumatoid arthritis [37], and atherosclerosis [38]. In CTSK knockout mice model of rheumatoid arthritis, not only the number of immune cells, but also the Toll-like receptors including TLR4, TLR5, and TLR9 showed a significant decrease. Consistently, the expression of CTSK is reduced in the atherosclerosis model of TLR4 knockout mice [38], indicating a close interaction between CTSK and TLR4. In this study, we verified a colocalization and interaction of CTSK and TLR4 using both immunofluorescence staining and Co-IP assay. Moreover, TLR4 is indispensable in CTSK-mediated recruitment of M2 macrophages, because TLR4 antibodies could totally block rCTSK-mediated M2 macrophages transition. Most importantly, the CTSK stimulated tumor growth and hepatic metastasis was totally abolished in TLR4 ${ }^{-l-}$ mice. Meanwhile, CTSK lost the stimulatory role of inducing M2 macrophages polarization in $\mathrm{TLR} 4^{-1-}$ mice.

As M2 macrophages promoted CRC growth and invasion mainly by releasing chemokines like IL17 and EGFR [39], we detected the secretion of 40 chemokines by TAMs before and after the treatment of CTSK. Secretion of inflammatory factors like IL4, IL10, IL17, as well as the expression of their receptors were significantly upregulated by the administration of CTSK. Further study confirmed the stimulatory role of those factors on CRC invasion and migration through a NFkB-dependent pathway. It is wellknown that the IL17 receptor (IL-17R) activates downstream NFKB via the transduction complex IL-17R-Act1TRAF6 [40]. In our study, IL10 and IL17 could promote the dissociation of IKK to release of IKB, and induce the following translocation of $\mathrm{p}-\mathrm{NF} \mathrm{NB}$ in the nucleus.

In summary, we identified CTSK as a vital mediator between the imbalance of intestinal microbiota and CRC metastasis. CRC-secreted CTSK stimulates CRC progression through accelerating M2 polarization of TAMs in a TLR4-mTOR-dependent pathway. Meanwhile, chemokines secreted by activated M2 macrophage, in turn, promote CRC cells invasion and metastasis by activating NFKB pathway. The current research illustrates a CTSK-mediatedpositive feedback loop between CRC and TAMs during metastasis (Fig. 8c), prompting CTSK as a novel predictive biomarker and feasible therapeutic target for CRC.

Funding This work was supported by the National Natural Science Foundation of China (Nos. 81572813, 81773082, 81702903, 81872423), Guangdong Natural Science Foundation (2017A030310038, 2018B030311036) and Fork Ying Tung Education Foundation (161035).

Author contributions LZ led study design and prepared the manuscript; RL, RZ, and HW carried out the experiments; W-DL, L-JX and M-XP performed statistical analysis; X-QY, W-QZ and S-BY assisted in tissue sample collection and clinical analysis; Y-QD performed data analysis and interpretation.

\section{Compliance with ethical standards}

Conflict of interest The authors declare that they have no conflict of interest.

Publisher's note: Springer Nature remains neutral with regard to jurisdictional claims in published maps and institutional affiliations.

\section{References}

1. De Rosa M, Pace U, Rega D, Costabile V, Duraturo F, Izzo P, et al. Genetics, diagnosis and management of colorectal cancer (Review). Oncol Rep. 2015;34:1087-96.

2. Joyce JA, Fearon DT. T cell exclusion, immune privilege, and the tumor microenvironment. Science. 2015;348:74-80.

3. Chung L, Orberg ET, Geis AL, Chan JL, Fu K, DeStefano Shields $\mathrm{CE}$, et al. Bacteroides fragilis toxin coordinates a pro-carcinogenic inflammatory cascade via targeting of colonic epithelial cells. Cell Host Microbe. 2018;23:421.

4. Dejea CM, Fathi P, Craig JM, Boleij A, Taddese R, Geis AL, et al. Patients with familial adenomatous polyposis harbor colonic biofilms containing tumorigenic bacteria. Science. 2018; 359:592-7.

5. Routy B, Le Chatelier E, Derosa L, Duong CPM, Alou MT, Daillere R, et al. Gut microbiome influences efficacy of PD-1based immunotherapy against epithelial tumors. Science. 2018;359:91-7.

6. Singh N, Gurav A, Sivaprakasam S, Brady E, Padia R, Shi H, et al. Activation of Gpr109a, receptor for niacin and the commensal metabolite butyrate, suppresses colonic inflammation and carcinogenesis. Immunity. 2014;40:128-39.

7. Rooks MG, Garrett WS. Gut microbiota, metabolites and host immunity. Nat Rev Immunol. 2016;16:341-52.

8. Novinec M, Lenarcic B. Cathepsin K: a unique collagenolytic cysteine peptidase. Biol Chem. 2013;394:1163-79.

9. Aguda AH, Panwar P, Du X, Nguyen NT, Brayer GD, Bromme D. Structural basis of collagen fiber degradation by cathepsin K. Proc Natl Acad Sci USA. 2014;111:17474-9.

10. Leusink FK, Koudounarakis E, Frank MH, Koole R, van Diest PJ, Willems SM. Cathepsin K associates with lymph node metastasis and poor prognosis in oral squamous cell carcinoma. Bmc Cancer. 2018;18:385.

11. Duong LT, Wesolowski GA, Leung P, Oballa R, Pickarski M. Efficacy of a cathepsin $\mathrm{K}$ inhibitor in a preclinical model for prevention and treatment of breast cancer bone metastasis. Mol Cancer Ther. 2014;13:2898-909.

12. Xu H, Ma Y, Zhang Y, Pan Z, Lu Y, Liu P, et al. Identification of cathepsin $\mathrm{K}$ in the peritoneal metastasis of ovarian carcinoma using in-silico, gene expression analysis. J Cancer. 2016;7:722-9.

13. Verbovsek U, Motaln H, Rotter A, Atai NA, Gruden K, Van Noorden CJ, et al. Expression analysis of all protease genes reveals cathepsin $\mathrm{K}$ to be overexpressed in glioblastoma. PLoS ONE. 2014;9:e111819.

14. Christensen J, Shastri VP. Matrix-metalloproteinase-9 is cleaved and activated by cathepsin K. BMC Res Notes. 2015;8:322.

15. Kleer CG, Bloushtain-Qimron N, Chen YH, Carrasco D, Hu M, Yao J, et al. Epithelial and stromal cathepsin K and CXCL14 expression in breast tumor progression. Clin Cancer Res. 2008;14:5357-67.

16. Zhao L, Wang H, Liu C, Liu Y, Wang X, Wang S, et al. Promotion of colorectal cancer growth and metastasis by the LIM and SH3 domain protein 1. Gut. 2010;59:1226-35. 
17. Cummins J, Cronin M, van Pijkeren JP, Gahan CG, Tangney M. Bacterial systems for gene delivery to systemic tumors. Methods Mol Biol. 2014;1141:201-9.

18. Hu J, Luo H, Wang J, Tang W, Lu J, Wu S, et al. Enteric dysbiosis-linked gut barrier disruption triggers early renal injury induced by chronic high salt feeding in mice. Exp Mol Med. 2017;49:e370.

19. Nimri L, Spivak O, Tal D, Schalling D, Peri I, Graeve L, et al. A recombinant fungal compound induces anti-proliferative and pro-apoptotic effects on colon cancer cells. Oncotarget. 2017;8:28854-64.

20. Zhang X, Goncalves R, Mosser DM. The isolation and characterization of murine macrophages. Curr Protoc Immunol. 2008; Chapter 14: Unit 14.1.

21. Liao Q, Li R, Zhou R, Pan Z, Xu L, Ding Y, et al. LIM kinase 1 interacts with myosin- 9 and alpha-actinin- 4 and promotes colorectal cancer progression. Br J Cancer. 2017;117:563-71.

22. Hirabara S, Kojima T, Takahashi N, Hanabayashi M, Ishiguro N. Hyaluronan inhibits TLR-4 dependent cathepsin K and matrix metalloproteinase 1 expression in human fibroblasts. Biochem Biophys Res Commun. 2013;430:519-22.

23. Caronni N, Savino B, Bonecchi R. Myeloid cells in cancer-related inflammation. Immunobiology. 2015;220:249-53.

24. Tilg H, Adolph TE, Gerner RR, Moschen AR. The intestinal microbiota in colorectal cancer. Cancer Cell. 2018;33:954-64.

25. Schulz MD, Atay C, Heringer J, Romrig FK, Schwitalla S, Aydin $\mathrm{B}$, et al. High-fat-diet-mediated dysbiosis promotes intestinal carcinogenesis independently of obesity. Nature. 2014;514:508-12.

26. Jorgensen SF, Troseid M, Kummen M, Anmarkrud JA, Michelsen $\mathrm{AE}$, Osnes LT, et al. Altered gut microbiota profile in common variable immunodeficiency associates with levels of lipopolysaccharide and markers of systemic immune activation. Mucosal Immunol. 2016;9:1455-65.

27. Cordes C, Bartling B, Simm A, Afar D, Lautenschlager C, Hansen $\mathrm{G}$, et al. Simultaneous expression of Cathepsins B and K in pulmonary adenocarcinomas and squamous cell carcinomas predicts poor recurrence-free and overall survival. Lung Cancer. 2009;64:79-85.

28. Herroon MK, Rajagurubandara E, Rudy DL, Chalasani A, Hardaway AL, Podgorski I. Macrophage cathepsin K promotes prostate tumor progression in bone. Oncogene. 2013;32:1580-93.

29. Kos J, Sekirnik A, Premzl A, Zavasnik Bergant V, Langerholc T, Turk B, et al. Carboxypeptidases cathepsins $\mathrm{X}$ and $\mathrm{B}$ display distinct protein profile in human cells and tissues. Exp Cell Res. 2005;306:103-13.

30. Guiet R, Van Goethem E, Cougoule C, Balor S, Valette A, Al Saati $\mathrm{T}$, et al. The process of macrophage migration promotes matrix metalloproteinase-independent invasion by tumor cells. J Immunol. 2011;187:3806-14.

31. Tan SY, Fan Y, Luo HS, Shen ZX, Guo Y, Zhao LJ. Prognostic significance of cell infiltrations of immunosurveillance in colorectal cancer. World J Gastroenterol. 2005;11:1210-4.

32. Cui YL, Li HK, Zhou HY, Zhang T, Li Q. Correlations of tumor-associated macrophage subtypes with liver metastases of colorectal cancer. Asian Pac J Cancer Prev. 2013;14:1003-7.

33. Duvel K, Yecies JL, Menon S, Raman P, Lipovsky AI, Souza AL, et al. Activation of a metabolic gene regulatory network downstream of mTOR complex 1. Mol Cell. 2010;39:171-83.

34. Salmiheimo AN, Mustonen HK, Vainionpaa SA, Shen Z, Kemppainen EA, Seppanen HE, et al. Increasing the inflammatory competence of macrophages with IL- 6 or with combination of IL4 and LPS restrains the invasiveness of pancreatic cancer cells. J Cancer. 2016;7:42-9.

35. Fan Y, Liu B. Expression of toll-like receptors in the mucosa of patients with ulcerative colitis. Exp Ther Med. 2015;9:1455-9.

36. Watanabe $\mathrm{T}$, Takahashi $\mathrm{N}$, Hirabara $\mathrm{S}$, Ishiguro $\mathrm{N}$, Kojima $\mathrm{T}$. Hyaluronan inhibits Tlr-4-dependent RANKL expression in human rheumatoid arthritis synovial fibroblasts. PLoS ONE. 2016;11:e0153142.

37. Donners MM, Bai L, Lutgens SP, Wijnands E, Johnson J, Schurgers LJ, et al. Cathepsin K deficiency prevents the aggravated vascular remodeling response to flow cessation in ApoE-/mice. PLoS ONE. 2016;11:e0162595.

38. Sun Y, Ishibashi M, Seimon T, Lee M, Sharma SM, Fitzgerald $\mathrm{KA}$, et al. Free cholesterol accumulation in macrophage membranes activates Toll-like receptors and p38 mitogen-activated protein kinase and induces cathepsin K. Circ Res. 2009;104:45565.

39. Popena I, Abols A, Saulite L, Pleiko K, Zandberga E, Jekabsons $\mathrm{K}$, et al. Effect of colorectal cancer-derived extracellular vesicles on the immunophenotype and cytokine secretion profile of monocytes and macrophages. Cell Commun Signal. 2018;16:17.

40. Gaffen SL. Structure and signalling in the IL-17 receptor family. Nat Rev Immunol. 2009;9:556-67. 